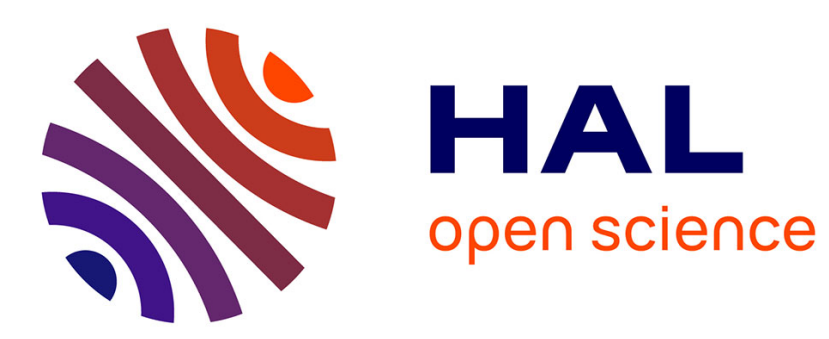

\title{
Exact solution for granular flows
}

Gloria Faccanoni, Anne Mangeney

\section{To cite this version:}

Gloria Faccanoni, Anne Mangeney. Exact solution for granular flows. International Journal for Numerical and Analytical Methods in Geomechanics, 2012, 37 (10), pp.1408-1433. 10.1002/nag.2124 . hal-00776614

\section{HAL Id: hal-00776614 https://hal.inria.fr/hal-00776614}

Submitted on 27 Feb 2020

HAL is a multi-disciplinary open access archive for the deposit and dissemination of scientific research documents, whether they are published or not. The documents may come from teaching and research institutions in France or abroad, or from public or private research centers.
L'archive ouverte pluridisciplinaire HAL, est destinée au dépôt et à la diffusion de documents scientifiques de niveau recherche, publiés ou non, émanant des établissements d'enseignement et de recherche français ou étrangers, des laboratoires publics ou privés. 


\title{
Exact solution for granular flows
}

\author{
Gloria Faccanoni $^{1 *}$, Anne Mangeney ${ }^{2,3}$ \\ ${ }^{1}$ Institut de mathématiques de Toulon et du Var, Université du Sud Toulon-Var, 83957 La Garde, France. \\ ${ }^{2}$ Équipe Sismologie, Institut de Physique du Globe de Paris-CNRS, 1 rue Jussieu, 75238 Paris, France. \\ ${ }^{3}$ University Paris Diderot 7, PRES Sorbonne Paris Cité.
}

\begin{abstract}
SUMMARY
In this paper we present the exact solution of the Riemann problem for the non-linear one-dimensional socalled shallow-water or Saint-Venant equations with friction proposed by SAVAGE and HUTTER to describe debris avalanches. This model is based on the depth-averaged thin layer approximation of granular flows over sloping beds and takes into account a Coulomb type friction law with a constant friction coefficient. A particular configuration of the Riemann problem corresponds to a dam of infinite length in one direction from which granular material is released from rest at a given time over an inclined rigid or erodible bed. We solve analytically and numerically the depth-averaged long-wave equations derived in a topographylinked coordinate system for all the possible Riemann problems. The detailed mathematical proof of the derivation of the analytical solutions and the analysis of their structure and properties is intended first of all for geophysicists, mathematicians and physicists because of the possible extension of this study to more complex problems (geometries, friction laws,...). The numerical solution of a first-order finitevolume method based on a Godunov-type scheme is compared with the proposed exact Riemann problem solution. This solution is used to solve the dam-break problem and analyze the influence of the thickness of the erodible bed on the speed of the granular front. Comparison with existing experimental results shows that, for an erodible bed, the equations lack fundamental physical significance to reproduce the observed dynamics of erosive granular flows. Copyright (c) 0000 John Wiley \& Sons, Ltd.
\end{abstract}

Received...

KEY WORDS: Shallow-water equations; Mohr-Coulomb type friction law; Analytical solution; Riemann problem; dam-break

\section{INTRODUCTION}

Analytical and numerical modeling of granular flows is a key issue for industrial and geophysical applications. Indeed, natural granular flows such as debris flows and landslides play a key role in erosion processes on the Earth's surface and represent one of the major natural hazards threatening life and property in mountainous, volcanic, seismic and coastal areas. Despite the large amount of work devoted to this problem, the mechanisms that govern flow dynamics and deposition in a natural environment are still unclear and key questions remain unanswered, for example concerning the origin of the high mobility of some natural granular flows (e.g. Crosta et al. [3, 4], Hungr and Evans [14], Iverson et al. [15], Mangeney [26], Mangeney et al. [29, 31], McDougall and Hungr [34, 35], Sovilla et al. [40, 41]).

Discrete element or continuum methods can be used to simulate natural granular flows. While discrete element methods may reproduce laboratory experiments quite accurately (see e.g. Lacaze

\footnotetext{
${ }^{*}$ Correspondence to: IMATH, Université du Sud Toulon-Var, 83957 La Garde, France.

E-mail: gloria.faccanonieuniv-tln.fr 
et al. [20]), they can handle only a limited number of tractable particles. This makes them unsuitable for natural flows where the observed broad size distribution with well-represented classes of different sizes involves many more particles than is computationally possible at the moment (e.g. Zhao et al. [47], Voivret et al. [46]). Similarly, solving the complete continuum 3D equations of granular mass motion with sufficient resolution to describe the real topography also leads to prohibitive computational costs. For this reason, most of the models dealing with natural flows are based on the so-called thin layer approximation (i.e. the thickness of the flowing mass is assumed to be small compared to its downslope extension). These models make use of a depth-averaged continuum description of the flow where the dissipation is described using Coulomb-type friction laws as first proposed by Savage and Hutter [39]. These assumptions lead to a system of hyperbolic equations for the thickness and slope-parallel, depth-averaged velocity field of the granular flow. These equations have been used to successfully reproduce a large range of experimental granular flows (e.g. Iverson et al. [15], Mangeney et al. [27], Mangeney-Castelnau et al. [32], Pouliquen and Forterre [37]) and natural landslides (Favreau et al. [8], Hibert et al. [12], Kuo et al. [19], Lucas and Mangeney [23], Lucas et al. [24], Pirulli and Mangeney [36]). Analytical solutions for this system of equations have provided insight into the scaling laws observed in experimental and natural granular flows (Hibert et al. [12], Kerswell [18], Lube et al. [22], Lucas and Mangeney [23], Mangeney et al. [29], Mangeney-Castelnau et al. [32], Roche et al. [38]).

However, the entrainment processes related to the presence of an erodible bed on the avalanche path is not or poorly taken into account in present analytical and numerical models. Entrainment of bed material along the slope has long been suspected to play a key role in flow dynamics, possibly driving landslides over unusually long distances (e.g. Hungr and Evans [14], Mangeney et al. [31]). Indeed, recent laboratory experiments and numerical simulations have shown that the presence of a very thin layer of erodible material lying on an inclined bed may increase the maximum runout distance of a granular avalanche flowing down the slope by up to $40 \%$ (Iverson et al. [15], Mangeney [26], Mangeney et al. [29]) and even generate granular surges that propagate at constant velocity along the slope (Mallogi et al. [25], Mangeney et al. [27], Pouliquen and Forterre [37]). Even though erosion/deposition processes in natural flows are expected to be very complex involving in particular segregation and recirculation processes (e.g. Gray and Ancey [11]), the question here is whether or not classical depth-averaged thin layer models can be used to obtain a first order approximation of the effect of the presence of an erodible bed on granular flow dynamics.

We will investigate here an analytical solution of the dam-break problem, when a granular mass is suddenly released from rest over an inclined plane covered by a thin erodible bed made of the same material. This configuration is similar to laboratory experiments performed by Mangeney et al. [29], designed to mimic erosion processes of natural flows traveling over deposits built up by earlier events (see Figure 1).

Exact solutions of dam-break problems were first developed for shallow-water equations (i.e. thin layer) without friction (see for example Trangenstein [44]). Dam-break problems are typical Riemann problems, i.e. initial value problems for conservation laws with initial data given by two constant states. Ritter (Stoker [42]) addressed the case of one-dimensional water floods created by dam failure over a horizontal plane without friction and over a dry bed. The case of the dam-break of a frictionless fluid over a horizontal wet bed has been dealt with by Stoker [42]. These two cases correspond to two different initial conditions of the same equations. All the possible Riemann problems corresponding to the flow of a frictionless fluid over a horizontal plane are described in several books (see for example Godlewski and Raviart [10], Trangenstein [44]), providing seven possible wave patterns (including the two cases mentioned above). Ungarish [45] solved the case of the release of a frictionless fluid mass into a frictionless fluid with a different density. Karelsky et al. $[16,17]$ extended the analytical solution of the Riemann problem to a frictionless fluid over a sloping bed. Mangeney et al. [28] generalized the Ritter solution to granular flows over a sloping dry rough plane described by the Savage-Hutter model (i.e. dissipation reduced to a Coulomb type friction law at the base of the flow). Karelsky et al. [16] and Mangeney et al. [28] showed that the solution for the fluid dam-break over an inclined bed differed from the solution of the classical shallow water equations because the characteristics of the system are branches of parabolas (and not straight lines) 


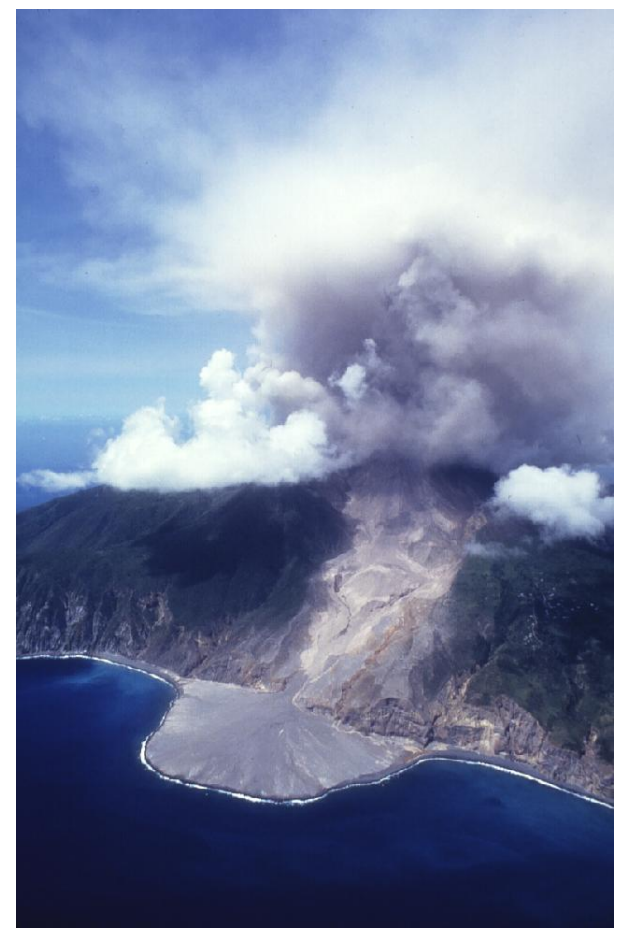

(a) Deposit from a $45 \mathrm{Mm}^{3}$ debris avalanche that occurred on December 24th 1997 in Montserrat, Lesser Antilles.

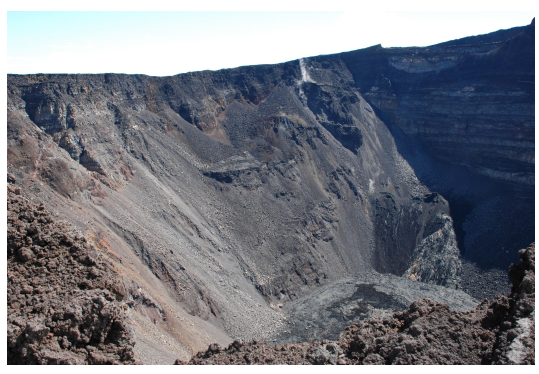

(b) Unconfined rockfalls in the Dolomieu crater, La Réunion with volumes in the range of 1 to $10^{3} \mathrm{~m}^{3}$ (Hibert et al. [12]).

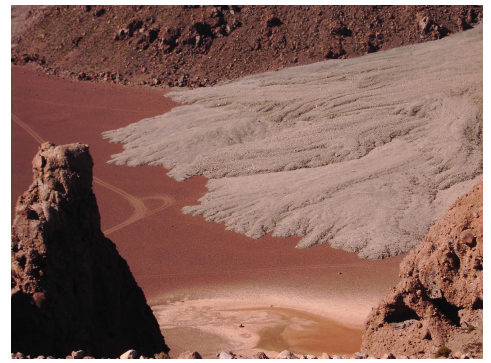

(c) Pyroclastic flows from the 1993 eruption of the Lascar Volcano, Chile.

Figure 1. Three examples of geophysical granular flows that traveled down slopes over the deposits of earlier events.

and the domain of constant flow is transformed into domains of uniformly accelerated flow. Exact (analytical or semi-analytical) solutions for the release of a finite initial mass over a rough bed with various geometries have been developed recently (Ancey et al. [1], Fernandez-Feria [9], Hogg [13], Kerswell [18]) for dam-break over a dry bed. For the case of the release of a finite rectangular mass over a dry horizontal bed, a double Riemann problem has to be solved (Kerswell [18]). When the slope of the released finite mass is not parallel to the slope of the plane, as in Ancey et al. [1] and Dressler [6], a more complicated Cauchy problem has to be solved instead of a Riemann problem. This involves a system of partial differential equations together with piecewise constant data exhibiting a single discontinuity.

The aim of the present paper is to compute the exact solution for the shallow-water equations over an inclined plane (with or without a Mohr-Coulomb type friction law at the base of the flow) for all possible Riemann problems, providing once again seven possible wave patterns, including the case of the vacuum (i.e. corresponding to a thickness of the granular mass $h=0$ ) in the initial data and the appearance of the vacuum at a later time. The objective is to provide mathematical proof of the derivation of the exact solution for all these cases, expressing the spatio-temporal solutions for the depth and velocity of the flow as well as the solution properties for direct use by geophysicists, physicists or mathematicians. A particular Riemann problem corresponds to the case of granular flow over a bed covered by a thin layer of granular material. For the case of an initial vacuum, we obtained the same result as Mangeney et al. [28], without an initial vacuum, we supporting the results of Mangeney et al. [29].

Although the solution treats idealized cases never strictly encountered in nature, these unsteady solutions may help reveal the asymptotic behavior of some real problems in certain limiting cases (e.g. Zhao et al. [48]). Because they are mathematically accurate, exact solutions provide important 
test cases (i.e. benchmark solutions) for numerical algorithms designed to integrate the depthaveraged thin layer equations for granular flows. This is particularly convenient for the purpose of obtaining an approximate or even an exact Riemann solver to be used eventually in numerical integration by means of a Godunov-type method (LeVeque [21], Toro [43]).

The paper is structured as follows. Section 2 presents the governing equations and the Riemann problem. Section 3 describes the complete set of exact solutions to a general Riemann problem. In section 4, we show comparisons between the exact solution and the numerical results obtained using the Rusanov scheme for all wave patterns. In Section 5, the exact solution is used to solve specific dam-break problems and to investigate the influence of the friction coefficient and the thickness of the erodible bed on the front position and velocity. The detailed proof of the derivation of this exact solution is presented in the Appendix. We describe the mathematical properties of the shallow water equations with a Mohr-Coulomb friction term written in a conservative form. This transformation makes it possible to remove the source term and work with an usual hyperbolic system of conservation laws. A detailed description of the construction of the analytical solution follows, presented in four steps: i) formulation of the Riemann problem as a system for the height of the material and for a modified velocity; ii) solution for rarefaction waves; iii) solution for shock waves; iv) combination with the previous study to solve the general Riemann problem. Finally we check that the total energy function is an entropy function for the system.

\section{GOVERNING EQUATIONS}

\subsection{Mass and momentum conservation}

The one-dimensional flow of an incompressible fluid is described by mass and momentum conservation equations. Gravitational flows are generally long and shallow so that the long-wave approximation along the slope is valid $(h \ll L$, where $h$ and $L$ are the thickness and typical extent of the flow along the slope, respectively). The equations are obtained by depth-averaging NavierStokes equations for an incompressible fluid and by using the free surface boundary condition. The coordinate system is linked to the topography: in our case the $x$-axis is parallel to the uniform slope with an angle $\varphi$ to the horizontal (see Figure 2). Following Savage and Hutter [39] and Mangeney et al. [28], a Mohr-Coulomb type friction law has been introduced in our model to describe debris or dense snow avalanches. Mass and momentum equations can be written as

$$
\left\{\begin{array}{l}
\partial_{t} h+\partial_{x}(h u)=0 \\
\partial_{t}(h u)+\partial_{x}\left(h u^{2}+g_{z} h^{2} / 2\right)=m h,
\end{array}\right.
$$

where $x \in \mathbb{R}$ is the downstream coordinate and $t>0$ is the time. The unknowns are $h \equiv h(x, t) \geq 0$, the flow depth measured perpendicular to the plane, and $u \equiv u(x, t) \in \mathbb{R}$, the depth-averaged flow velocity. The other terms are $g_{z} \equiv g \cos (\varphi)$, the projection of the gravitational acceleration on the vertical axis (perpendicular to the plane), and $m \equiv g_{x}+F=g_{z}(\tan (\varphi)-\tan (\delta))$, the constant $x$ acceleration resulting from the sum of the forces due to gravity and friction. The friction force is the well-known Coulomb-type friction law proposed by Savage and Hutter [39] to describe granular flow behavior, $F=-g_{z} \tan (\delta) \leq 0$, where $\delta$ is the basal friction angle. Here we assume $\tan (\varphi) \geq \tan (\delta)$, so that, when initially flowing, the granular mass never stops on the plane. If $m \geq 0$ and $u \geq 0$, the system can represent for example a granular flow over an inclined plane covered or not by a layer of material made of the same material as the flowing granular mass.

\subsection{Initial conditions: the Riemann problem}

We consider a Riemann problem, i.e. the system (1) with particular initial data consisting of two constant states separated by a single discontinuity:

$$
(h, u)(x, t=0)= \begin{cases}\left(h_{L}, u_{L}\right), & \text { if } x<0, \\ \left(h_{R}, u_{R}\right), & \text { if } x>0,\end{cases}
$$




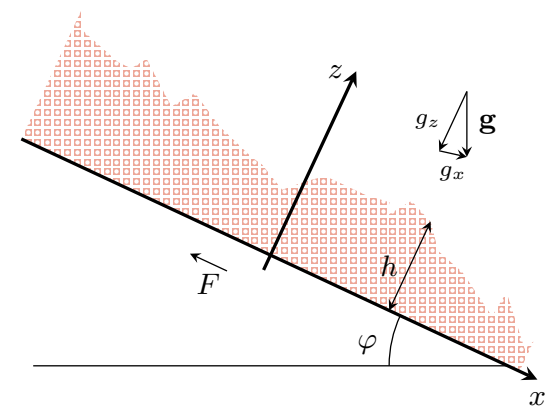

Figure 2. Geometry of the granular flow and reference frame

where $h_{L} \geq 0, u_{L} \in \mathbb{R}, h_{R} \geq 0$ and $u_{R} \in \mathbb{R}$ are four constants. For example, the instantaneous release of fluid from rest, typical of natural gravitational flows, leads to a significant initial discontinuity that corresponds to a Riemann problem. A dam with infinite length in one direction where material is released from rest corresponds to $h_{L}>h_{R}=0$ and $u_{L}=u_{R}=0$ (see Figure 7a). Granular material flowing over an inclined plane covered by an erodible bed where material is released from rest corresponds to $h_{L}>h_{R}>0$ and $u_{L}=u_{R}=0$ (see Figure 7b). The solution of these problems has a relatively simple structure and can be calculated explicitly.

Unrealistic as it may seem (unbounded domain and piecewise constant initial data with a single discontinuity), the solution to the Riemann problem is extremely valuable for practical applications. Many laboratory experiments reproduce in fact the conditions of the Riemann problem. The solution to the Riemann problem also provides information on the structure of the system of equations and can be used as the building block for obtaining solutions to problems with more complex initial conditions. Moreover, many interesting test problems for numerical methods arise from onedimensional Riemann problems. This is particularly convenient for the purpose of obtaining an approximate or even an exact Riemann solver to be used eventually in the numerical integration of the equations (1) with any initial conditions by means of a Godunov-type method (LeVeque [21], Toro [43]). Indeed, in a numerical method, a set of discrete values $\left(h_{i}^{n}, u_{i}^{n}\right)$ is computed, presumably an approximation of $(h, u)\left(x_{i}, t^{n}\right)$ on a given set of grid points $\left\{\left(x_{i}, t^{n}\right)\right\}$, where $n$ denotes the time increment. A great deal of information on the local structure of the solution near $\left(x_{i}, t^{n}\right)$ is obtained by solving a Riemann problem with data $\left(h_{L}, u_{L}\right)=\left(h_{i}^{n}, u_{i}^{n}\right)$ and $\left(h_{R}, u_{R}\right)=$ $\left(h_{i+1}^{n}, u_{i+1}^{n}\right)$. Many numerical methods make use of these Riemann solutions.

\section{EXACT SOLUTION OF THE RIEMANN PROBLEM}

We present here the solutions of all possible Riemann problems (2) for the system (1). The detailed derivation of the exact solution is presented in the appendix for different initial conditions. Here we present and analyze the characteristics and properties of this exact solution. Neglecting the case of the vacuum (i.e. $h>0$ ), the structure of the solutions involves two distinct waves separating three regions. Across a wave, we can observe a discontinuity of the state variables, called a shock wave, or all variables may be continuous and there is a smooth transition, called a rarefaction wave or fan.

\subsection{The solution}

The solution consists of at most three states where $h$ and $u-m t$ are constant (the left, intermediate and right states, hereafter referred to as $L, *, R$, respectively) separated by rarefaction waves (where $h$ and $u$ are continuous) or admissible shock waves (where $h$ and $u$ exhibit a jump), see Figures 34. The wave separating the state $\left(h_{L}, u_{L}+m t\right)$ and the state $\left(h_{*}, u_{*}+m t\right)$ is called a 1-wave and can be a 1-rarefaction or a 1-shock wave. Similarly the wave separating the state $\left(h_{*}, u_{*}+m t\right)$ and the state $\left(h_{R}, u_{R}+m t\right)$ is called a 2-wave and can be a 2-rarefaction or a 2-shock wave. As for the shallow water system, the free surface $h(x, t)$ in the rarefaction waves is curved with a 


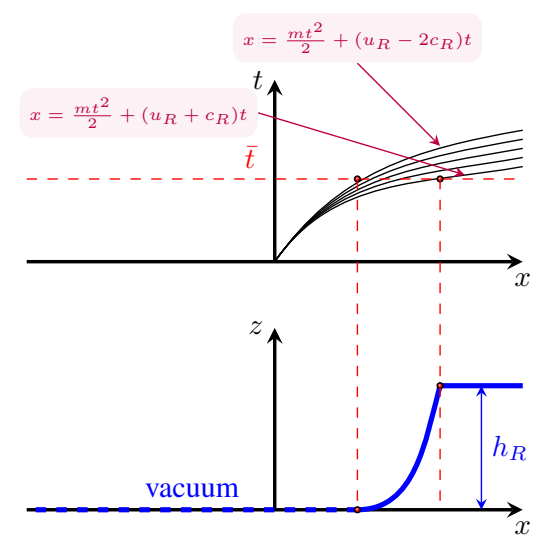

(a) Case 1.1: wave structure (top) and freesurface $h(x, t)$ for $t=\bar{t}$ (bottom)

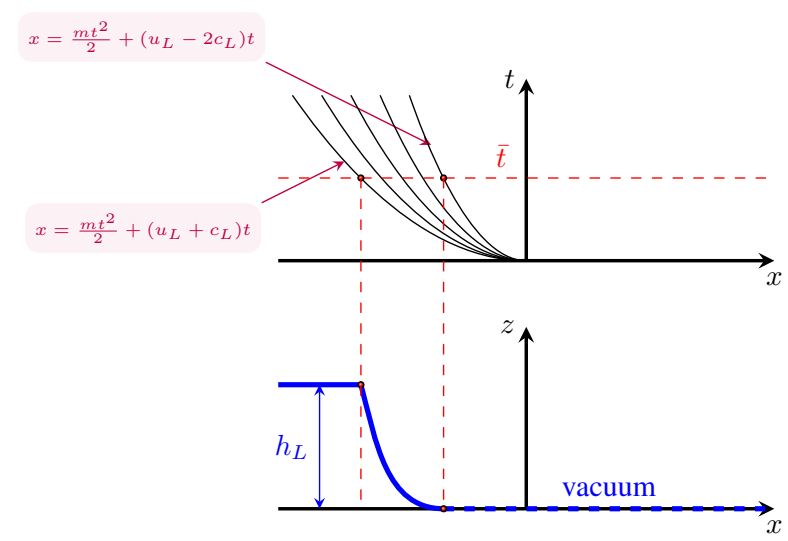

(b) Case 1.2: wave structure (top) and free-surface $h(x, t)$ for $t=\bar{t}$ (bottom)

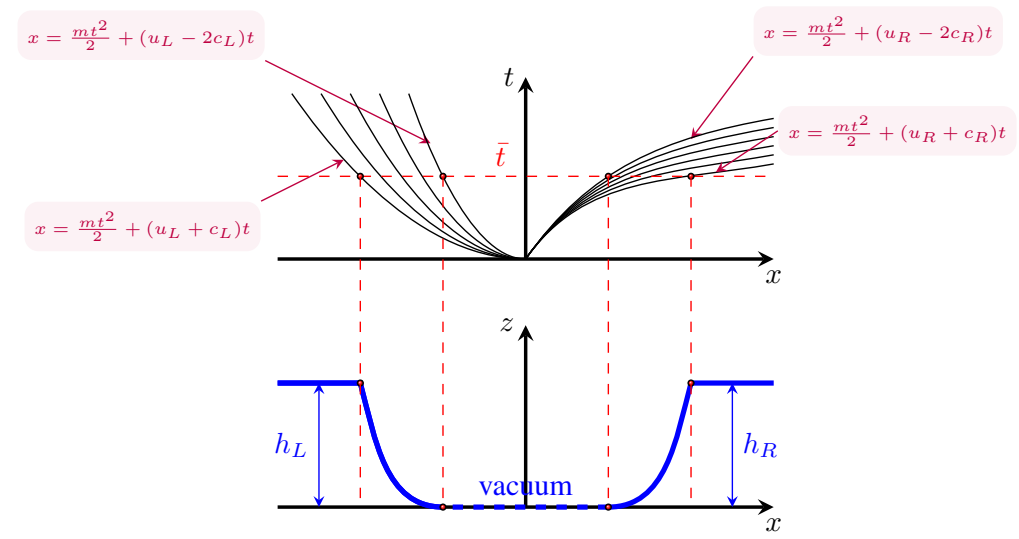

(c) Case 1.3: wave structure (top) and free-surface $h(x, t)$ for $t=\bar{t}$ (bottom)

Figure 3. Scheme of the solution in presence of a vacuum, i.e. zones where $h=0$ (type 1).

parabolic shape. If there is a vacuum region between two zones (between the left zone $L$ and the intermediate zone $*$ for a 1-rarefaction or between the intermediate zone $*$ and the right zone $R$ for a 2-rarefaction), the parabola has a horizontal tangent. On the contrary, if there is no vacuum region between the two zones, the slope of the free surface is discontinuous at these points.

Let us summarize here the complete solution of the system (1) with an initial condition of type (2) for all $m \in \mathbb{R}$ that has been derived using the method detailed in the Appendix. Depending on the initial conditions, different solutions are obtained that can be separated into two types. For the first type, the solution is analytical and includes a "dry" state or "vacuum" where the thickness of the flow is zero (cases 1.1-1.3). For the second type, the solution is semi-analytical ${ }^{\dagger}$ and involves only so-called "wet" states, i.e. the flow thickness is always strictly positive (cases 2.1-2.4).

We introduce the wave celerities $c_{L} \equiv \sqrt{g_{z} h_{L}}$ and $c_{R} \equiv \sqrt{g_{z} h_{R}}$.

Type 1. In the following three cases, the solution is analytical and contains regions where the thickness of the flow is zero. If the thickness $h=0$, the value of the velocity $u$ does not have any physical significance.

\footnotetext{
†Our solution is called semi-analytical rather than analytical because it depends on the solution (in general only numerical) of a nonlinear equation, here equation (3).
} 
1.1. If $h_{L}=0$, it is natural to consider $u_{L}=0$. The solution is composed of a 2-rarefaction wave as in Figure 3a. The flow-depth satisfies

$$
h(x, t)= \begin{cases}0, & \text { if } x-\frac{m t^{2}}{2} \leq\left(u_{R}-2 c_{R}\right) t, \\ \frac{\left(-u_{R}+2 c_{R}+\left(\frac{x}{t}-\frac{m t}{2}\right)\right)^{2}}{9 g_{z}} & \text { if }\left(u_{R}-2 c_{R}\right) t<x-\frac{m t^{2}}{2} \leq\left(u_{R}+c_{R}\right) t, \\ h_{R}, & \text { if } x-\frac{m t^{2}}{2}>\left(u_{R}+c_{R}\right) t,\end{cases}
$$

and the depth-averaged flow velocity satisfies

$$
u(x, t)= \begin{cases}m t, & \text { if } x-\frac{m t^{2}}{2}<\left(u_{R}-2 c_{R}\right) t, \\ \frac{u_{R}-2 c_{R}+2\left(\frac{x}{t}-\frac{m t}{2}\right)}{3}+m t & \text { if }\left(u_{R}-2 c_{R}\right) t<x-\frac{m t^{2}}{2} \leq\left(u_{R}+c_{R}\right) t, \\ u_{R}+m t, & \text { if } x-\frac{m t^{2}}{2}>\left(u_{R}-c_{R}\right) t .\end{cases}
$$

Note that when $x \rightarrow\left(m t^{2} / 2+\left(u_{R}-2 c_{R}\right) t\right)^{+}$, the flow depth is continuous because $h(x, t) \rightarrow 0$ but the depth-averaged velocity can be discontinuous because $u(x, t) \rightarrow$ $\left(u_{R}-2 c_{R}+m t\right)$.

1.2. If $h_{R}=0$, it is natural to consider $u_{R}=0$. The solution is composed of a 1-rarefaction wave as in Figure 3b. The flow-depth satisfies

$$
h(x, t)= \begin{cases}h_{L}, & \text { if } x-\frac{m t^{2}}{2} \leq\left(u_{L}-c_{L}\right) t, \\ \frac{\left(u_{L}+2 c_{L}-\left(\frac{x}{t}-\frac{m t}{2}\right)\right)^{2}}{9 g_{z}}, & \text { if }\left(u_{L}-c_{L}\right) t<-\frac{m t^{2}}{2} \leq\left(u_{L}+2 c_{L}\right) t, \\ 0, & \text { if } x-\frac{m t^{2}}{2}>\left(u_{L}+2 c_{L}\right) t,\end{cases}
$$

and the depth-averaged flow velocity satisfies

$$
u(x, t)= \begin{cases}u_{L}+m t, & \text { if } x-\frac{m t^{2}}{2} \leq\left(u_{L}-c_{L}\right) t, \\ \frac{u_{L}+2 c_{L}+2\left(\frac{x}{t}-\frac{m t}{2}\right)}{3}+m t, & \text { if }\left(u_{L}-c_{L}\right) t<x-\frac{m t^{2}}{2} \leq\left(u_{L}+2 c_{L}\right) t, \\ m t, & \text { if } x-\frac{m t^{2}}{2}>\left(u_{L}+2 c_{L}\right) t .\end{cases}
$$

Note that when $x \rightarrow\left(m t^{2} / 2+\left(u_{L}+2 c_{L}\right) t\right)^{-}$, the flow depth is continuous because $h(x, t) \rightarrow 0$ but the depth-averaged velocity can be discontinuous because $u(x, t) \rightarrow$ $\left(u_{L}+2 c_{L}+m t\right)$.

1.3. If $h_{L}>0, h_{R}>0$ and $u_{R}-u_{L} \geq 2\left(c_{R}+c_{L}\right)$, the solution is composed of a 1rarefaction wave followed by a 2 -rarefaction wave separating a dry zone as in Figure $3 \mathrm{c}$. The flow-depth satisfies

$$
h(x, t)= \begin{cases}\frac{h_{L},}{\frac{\left(u_{L}+2 c_{L}-\left(\frac{x}{t}-\frac{m t}{2}\right)\right)^{2}}{9 g_{z}},}, & \text { if } x-\frac{m t^{2}}{2} \leq\left(u_{L}-c_{L}\right) t, \\ 0, & \text { if }\left(u_{L}-c_{L}\right) t<x-\frac{m t^{2}}{2} \leq\left(u_{L}+2 c_{L}\right) t, \\ \frac{\left(-u_{R}+2 c_{R}+\left(\frac{x}{t}-\frac{m t}{2}\right)\right)^{2}}{9 g_{z}}, & \text { if }\left(u_{L}+2 c_{L}\right) t<x-\frac{m t^{2}}{2} \leq\left(u_{R}-2 c_{R}\right) t, \\ h_{R}, & \text { if }\left(u_{R}-2 c_{R}\right) t<x-\frac{m t^{2}}{2} \leq\left(u_{R}+c_{R}\right) t,\end{cases}
$$

and the depth-averaged flow velocity satisfies

$$
u(x, t)= \begin{cases}u_{L}+m t, & \text { if } x-\frac{m t^{2}}{2} \leq\left(u_{L}-c_{L}\right) t, \\ \frac{u_{L}+2 c_{L}+2\left(\frac{x}{t}-\frac{m t}{2}\right)}{3}+m t & \text { if }\left(u_{L}-c_{L}\right) t<x-\frac{m t^{2}}{2} \leq\left(u_{L}+2 c_{L}\right) t, \\ m t, & \text { if }\left(u_{L}+2 c_{L}\right) t<x-\frac{m t^{2}}{2} \leq\left(u_{R}-2 c_{R}\right) t, \\ \frac{u_{R}-2 c_{R}+2\left(\frac{x}{t}-\frac{m t}{2}\right)}{3}+m t & \text { if }\left(u_{R}-2 c_{R}\right) t<x-\frac{m t^{2}}{2} \leq\left(u_{R}+c_{R}\right) t, \\ u_{R}+m t, & \text { if } x-\frac{m t^{2}}{2}>\left(u_{R}+c_{R}\right) t .\end{cases}
$$

Note that the flow depth and depth-averaged velocity are continuous throughout the domain . 


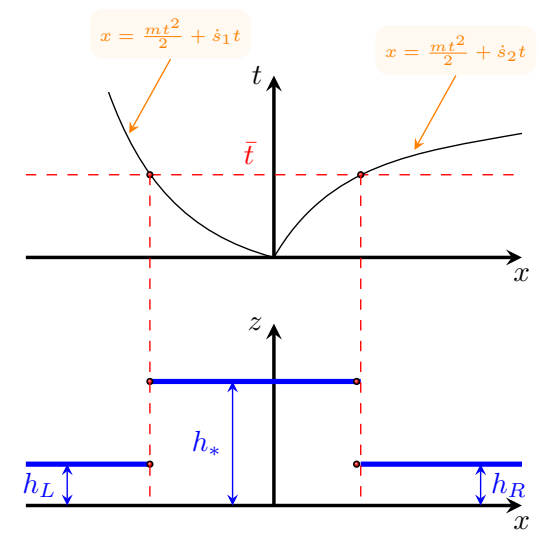

(a) Case 2.1: wave structure (top) and freesurface $h(x, t)$ for $t=\bar{t}$ (bottom)

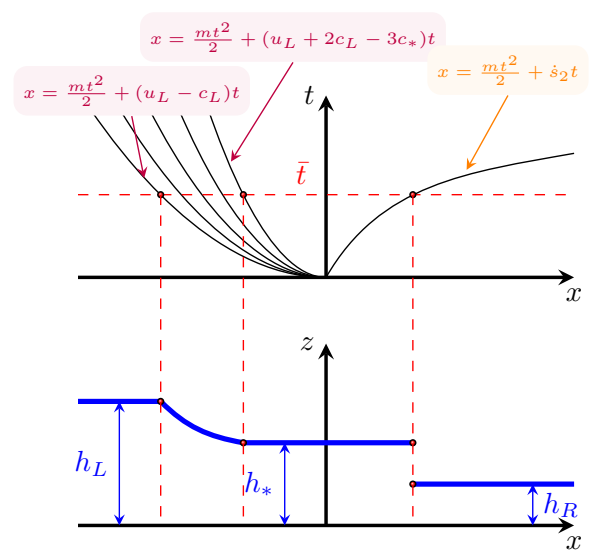

(c) Case 2.2: wave structure (top) and free-surface $h(x, t)$ for $t=\bar{t}$ (bottom)

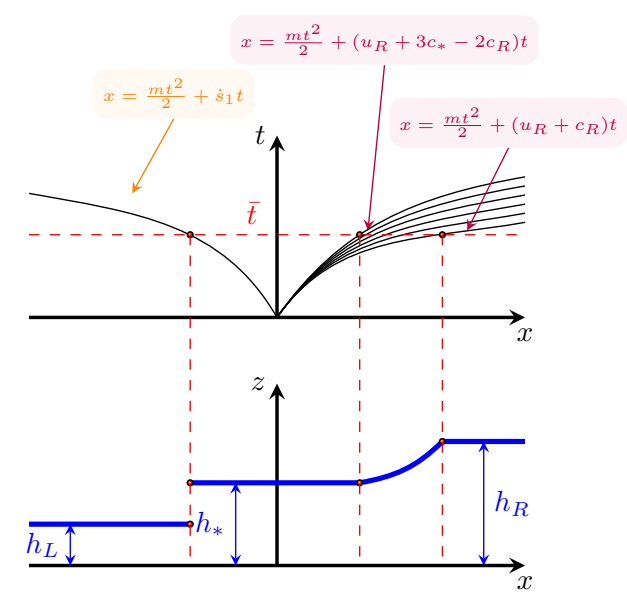

(b) Case 2.3: wave structure (top) and free-surface $h(x, t)$ for $t=\bar{t}$ (bottom)

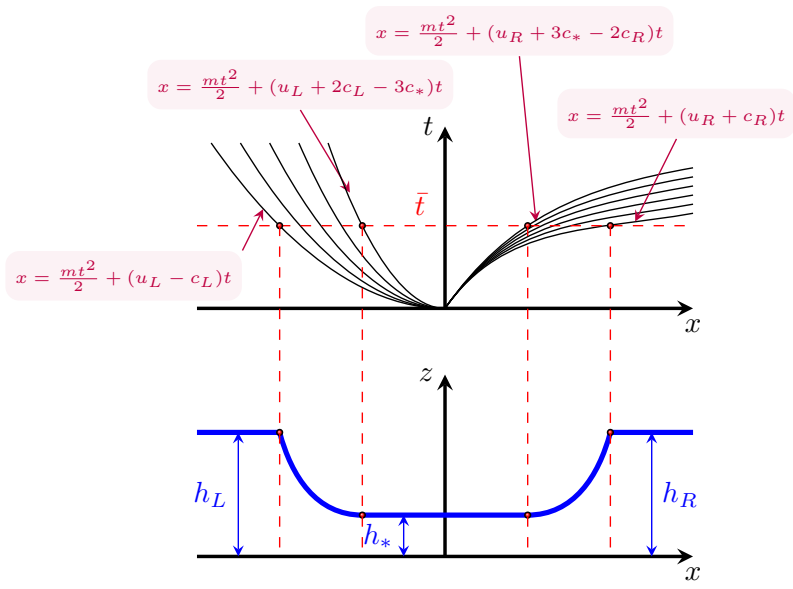

(d) Case 2.4: wave structure (top) and free-surface $h(x, t)$ for $t=\bar{t}$ (bottom)

Figure 4. Scheme of the solution without vacuum, i.e. $h>0$ for all $(x, t)$ (type 2).

Type 2. If $h_{L}>0, h_{R}>0$ and $u_{R}-u_{L}<2\left(c_{R}+c_{L}\right)$, we can compute semi-analytical solutions. We define $h_{*}$ as the root of the function

$$
f(h)=u_{R}-u_{L}+\left(h-h_{L}\right) z\left(h, h_{L}\right)+\left(h-h_{R}\right) z\left(h, h_{R}\right)
$$

with

$$
z(h, \psi)= \begin{cases}\frac{2 \sqrt{g_{z}}}{\sqrt{h}+\sqrt{\psi}} & \text { if } h \leq \psi \\ \sqrt{\frac{g_{z}}{2} \frac{h+\psi}{h \psi}} & \text { otherwise }\end{cases}
$$

and we note $u_{*} \equiv u_{R}+\left(h_{*}-h_{R}\right) z\left(h_{*}, h_{R}\right)$ and $c_{*} \equiv \sqrt{g_{z} h_{*}}$. Then we have the following four configurations. 
2.1. If $h_{*}>\max \left\{h_{L}, h_{R}\right\}$, the solution is composed of a 1-shock wave followed by a 2shock wave as in Figure 4a. The flow-depth satisfies

$$
h(x, t)= \begin{cases}h_{L}, & \text { if } x-\frac{m t^{2}}{2}<\dot{s}_{1} t, \\ h_{*}, & \text { if } \dot{s}_{1} t<x-\frac{m t^{2}}{2}<\dot{s}_{2} t, \\ h_{R}, & \text { if } x-\frac{m t^{2}}{2}>\dot{s}_{2} t,\end{cases}
$$

and the depth-averaged flow velocity satisfies

$$
u(x, t)= \begin{cases}u_{L}+m t, & \text { if } x-\frac{m t^{2}}{2}<\dot{s}_{1} t \\ u_{*}+m t, & \text { if } \dot{s}_{1} t<-\frac{m t^{2}}{2}<\dot{s}_{2} t, \\ u_{R}+m t, & \text { if } x-\frac{m t^{2}}{2}>\dot{s}_{2} t\end{cases}
$$

where the constant speeds of the discontinuities are

$$
\dot{s}_{1} \equiv u_{L}-h_{*} \sqrt{\frac{g_{z}}{2} \frac{h_{L}+h_{*}}{h_{L} h_{*}}}, \quad \dot{s}_{2} \equiv u_{R}+h_{*} \sqrt{\frac{g_{z}}{2} \frac{h_{*}+h_{R}}{h_{*} h_{R}}} .
$$

2.2. If $h_{L}<h_{*}<h_{R}$, the solution is composed of a 1-rarefaction wave followed by a 2shock wave as in Figure 4c. The flow-depth satisfies

$$
h(x, t)= \begin{cases}h_{L}, & \text { if } x-\frac{m t^{2}}{2}<\dot{s}_{1} t, \\ h_{*}, & \text { if } \dot{s}_{1} t<x-\frac{m t^{2}}{2} \leq\left(u_{R}+3 c_{*}-2 c_{R}\right) t, \\ \frac{\left(-u_{R}+2 c_{R}+\left(\frac{x}{t}-\frac{m t}{2}\right)\right)^{2}}{9 g_{z}} & \text { if }\left(u_{R}+3 c_{*}-2 c_{R}\right) t<x-\frac{m t^{2}}{2} \leq\left(u_{R}+c_{R}\right) t, \\ h_{R}, & \text { if } x-\frac{m t^{2}}{2}>\left(u_{R}+c_{R}\right) t,\end{cases}
$$

and the depth-averaged flow velocity satisfies

$$
u(x, t)= \begin{cases}u_{L}+m t, & \text { if } x-\frac{m t^{2}}{2}<\dot{s}_{1} t \\ u_{*}+m t, & \text { if } \dot{s}_{1} t<x-\frac{m t^{2}}{2} \leq\left(u_{R}+3 c_{*}-2 c_{R}\right) t, \\ \frac{u_{R}-2 c_{R}+2\left(\frac{x}{t}-\frac{m t}{2}\right)}{3}+m t & \text { if }\left(u_{R}+3 c_{*}-2 c_{R}\right) t<x-\frac{m t^{2}}{2} \leq\left(u_{R}+c_{R}\right) t, \\ u_{R}+m t, & \text { if } x-\frac{m t^{2}}{2}>\left(u_{R}+c_{R}\right) t,\end{cases}
$$

where the constant speed of the discontinuity is

$$
\dot{s}_{1} \equiv u_{L}-h_{*} \sqrt{\frac{g_{z}}{2} \frac{h_{L}+h_{*}}{h_{L} h_{*}}} .
$$

2.3. If $h_{L}>h_{*}>h_{R}$, the solution is composed of a 1-shock wave followed by a 2 rarefaction wave as in Figure 4b. The flow-depth satisfies

$$
h(x, t)= \begin{cases}h_{L}, & \text { if } x-\frac{m t^{2}}{2} \leq\left(u_{L}-c_{L}\right) t, \\ \frac{\left(u_{L}+2 c_{L}-\left(\frac{x}{t}-\frac{m t}{2}\right)\right)^{2}}{9 g_{z}}, & \text { if }\left(u_{L}-c_{L}\right) t<-\frac{m t^{2}}{2} \leq\left(u_{L}+2 c_{L}-3 c_{*}\right) t, \\ h_{*}, & \text { if }\left(u_{L}+2 c_{L}-3 c_{*}\right) t<x-\frac{m t^{2}}{2}<\dot{s}_{2} t, \\ h_{R}, & \text { if } x-\frac{m t^{2}}{2}>\dot{s}_{2} t,\end{cases}
$$

and the depth-averaged flow velocity satisfies

$$
u(x, t)= \begin{cases}u_{L}+m t, & \text { if } x-\frac{m t^{2}}{2} \leq\left(u_{L}-c_{L}\right) t, \\ \frac{u_{L}+2 c_{L}+2\left(\frac{x}{t}-\frac{m t}{2}\right)}{3}+m t, & \text { if }\left(u_{L}-c_{L}\right) t<x-\frac{m t^{2}}{2} \leq\left(u_{L}+2 c_{L}-3 c_{*}\right) t, \\ u_{*}+m t, & \text { if }\left(u_{L}+2 c_{L}-3 c_{*}\right) t<x-\frac{m t^{2}}{2}<\dot{s}_{2} t, \\ u_{R}+m t, & \text { if } x-\frac{m t^{2}}{2}>\dot{s}_{2} t,\end{cases}
$$

where the constant speed of the discontinuity is

$$
\dot{s}_{2} \equiv u_{R}+h_{*} \sqrt{\frac{g_{z}}{2} \frac{h_{*}+h_{R}}{h_{*} h_{R}}} .
$$


2.4. If $h_{*}<\min \left\{h_{L}, h_{R}\right\}$, the solution is composed of a 1-rarefaction wave followed by a 2-rarefaction wave as in Figure 4d. The flow-depth satisfies

$$
h(x, t)= \begin{cases}\frac{h_{L},}{\left(u_{L}+2 c_{L}-\left(\frac{x}{t}-\frac{m t}{2}\right)\right)^{2}} & \text { if } x-\frac{m t^{2}}{2} \leq\left(u_{L}-c_{L}\right) t, \\ 9 g_{z} & \text { if }\left(u_{L}-c_{L}\right) t<x-\frac{m t^{2}}{2} \leq\left(u_{L}+2 c_{L}-3 c_{*}\right) t, \\ h_{*}, & \text { if }\left(u_{L}+2 c_{L}-3 c_{*}\right) t<x-\frac{m t^{2}}{2} \leq\left(u_{R}+3 c_{*}-2 c_{R}\right) t, \\ \frac{\left(-u_{R}+2 c_{R}+\left(\frac{x}{t}-\frac{m t}{2}\right)\right)^{2}}{9 g_{z}}, & \text { if }\left(u_{R}+3 c_{*}-2 c_{R}\right) t<x-\frac{m t^{2}}{2} \leq\left(u_{R}+c_{R}\right) t, \\ h_{R}, & \text { if } x-\frac{m t^{2}}{2}>\left(u_{R}+c_{R}\right) t,\end{cases}
$$

and the depth-averaged flow velocity satisfies

$$
u(x, t)= \begin{cases}u_{L}+m t, & \text { if } x-\frac{m t^{2}}{2} \leq\left(u_{L}-c_{L}\right) t, \\ \frac{u_{L}+2 c_{L}+2\left(\frac{x}{t}-\frac{m t}{2}\right)}{3}+m t & \text { if }\left(u_{L}-c_{L}\right) t<x-\frac{m t^{2}}{2} \leq\left(u_{L}+2 c_{L}-3 c_{*}\right) t, \\ u_{*}+m t, & \text { if }\left(u_{L}+2 c_{L}-3 c_{*}\right) t<x-\frac{m t^{2}}{2} \leq\left(u_{R}+3 c_{*}-2 c_{R}\right) t, \\ \frac{u_{R}-2 c_{R}+2\left(\frac{x}{t}-\frac{m t}{2}\right)}{3}+m t & \text { if }\left(u_{R}+3 c_{*}-2 c_{R}\right) t<x-\frac{m t^{2}}{2} \leq\left(u_{R}+c_{R}\right) t, \\ u_{R}+m t, & \text { if } x-\frac{m t^{2}}{2}>\left(u_{R}+c_{R}\right) t .\end{cases}
$$

Remark. It is interesting to observe that this solution can be obtained from the solution of the classic shallow-water equations (Trangenstein [44]) with $m=0$ (i.e., the horizontal problem with $g=g_{z}$ ) by the change of reference frame: $X \equiv x-m t^{2} / 2, U \equiv u-m t$, where $m$ is the acceleration of the reference frame, $X$ and $U$ are the horizontal coordinate and velocity in the new reference frame (i.e. horizontal/vertical), respectively. Therefore, the Ritter's solution corresponds to cases 1.1 and 1.2 and the Stoker's solution to cases 2.2 and 2.3. We obtain the same change of variables as suggested by Karelsky et al. $[16,17]$ to generalize the classic shallow-water system to the case of flows over an uniform slope without friction, i.e. when $m \equiv g_{x}$.

\section{NUMERICAL TESTS}

Let us compare our exact solution with the numerical solution in all possible configurations. In order to represent all possible combinations of wave patterns, seven Riemann problems have been solved. The corresponding initial data are shown in Table I. We consider an inclined bottom of slope $\varphi=22^{\circ}$ and a friction angle $\delta=21^{\circ}$. Acceleration due to gravity is $g=9.81 \mathrm{~m} \mathrm{~s}^{-2}$, so that $m \simeq 0.1839 \mathrm{~m} \mathrm{~s}^{-2}$ (the flow is accelerated). In all cases, the initial discontinuity is located at $x=0 \mathrm{~m}$. When it is necessary to compute the intermediate state $\left(h_{*}, u_{*}\right)$, i.e. for tests (4)-(7), the proposed Riemann solver has been implemented using a Newton iterative method, with a stop criterion when the increment of two iterates or when $f(h)$ is $<10^{-10}$ (the function $f$ is defined in equation (3)). The initial guess for $h$ is taken to be the average of $h$ on the left and right states of the Riemann problem.

For comparison we report the solution of the same Riemann problems calculated with the firstorder Rusanov scheme (see for example Trangenstein [44, p. 179]) applied to solve the system (1) in the conservative form (5) (see Appendix) using a uniform grid of 5000 cells. Figure 5 shows the projection of the flow-depth $h(x, t)$ on the plane $(x, t)$ illustrating the wave patterns. The color scale represents the thickness of the granular mass. We obtain the theoretical wave structure of Figures 3 and 4: the waves have a parabolic shape, contrary to the classic shallow-water system where the waves are straight lines. In Figure 6 we observe that the numerical solution agrees very well with our exact solution. In particular, positions and types of all waves coincide, showing a very good agreement between the exact and numerical solution. ${ }^{\ddagger}$.

\footnotetext{
¥The spurious over(under) shoots in the numerical solution near the step position for the depth-averaged velocity in test (3) disappear at convergence. Similarly, the numerical velocity near the front in cases (1) and (2) converges toward the analytical solution when the grid is refined.
} 


\begin{tabular}{|c|c|c|c|}
\hline & Waves patterns & Left & Right \\
\hline Test (1) & $\begin{array}{l}\text { Case 1.1: 2-rarefaction } \\
\text { (Dam-break type problem, "dry bed") }\end{array}$ & $\begin{array}{l}h_{L}=0.0 \mathrm{~m} \\
u_{L}=0.0 \mathrm{~m} \mathrm{~s}^{-1}\end{array}$ & $\begin{array}{l}h_{R}=0.1446 \mathrm{~m} \\
u_{R}=0.0 \mathrm{~m} \mathrm{~s}^{-1}\end{array}$ \\
\hline Test (2) & $\begin{array}{l}\text { Case 1.2: 1-rarefaction } \\
\text { (Dam-break type problem, "dry bed") }\end{array}$ & $\begin{array}{l}h_{L}=0.1446 \mathrm{~m} \\
u_{L}=0.0 \mathrm{~m} \mathrm{~s}^{-1}\end{array}$ & $\begin{array}{l}h_{R}=0.0 \mathrm{~m} \\
u_{R}=0.0 \mathrm{~m} \mathrm{~s}^{-1}\end{array}$ \\
\hline Test (3) & $\begin{array}{l}\text { Case 1.3: 1-rarefaction 2-rarefaction } \\
\text { (with formation of a "dry zone") }\end{array}$ & $\begin{array}{l}h_{L}=0.0046 \mathrm{~m} \\
u_{L}=-1.0 \mathrm{~m} \mathrm{~s}^{-1}\end{array}$ & $\begin{array}{l}h_{R}=0.0046 \mathrm{~m} \\
u_{R}=1.0 \mathrm{~m} \mathrm{~s}^{-1}\end{array}$ \\
\hline Test (4) & Case 2.1: 1-shock 2-shock & $\begin{array}{l}h_{L}=0.1446 \mathrm{~m} \\
u_{L}=1.0 \mathrm{~m} \mathrm{~s}^{-1}\end{array}$ & $\begin{array}{l}h_{R}=0.1446 \mathrm{~m} \\
u_{R}=-1.0 \mathrm{~m} \mathrm{~s}^{-1}\end{array}$ \\
\hline Test (5) & $\begin{array}{l}\text { Case 2.2: 1-shock 2-rarefaction } \\
\text { (Dam-break type problem, "wet bed") }\end{array}$ & $\begin{array}{l}h_{L}=0.0046 \mathrm{~m} \\
u_{L}=0.0 \mathrm{~m} \mathrm{~s}^{-1}\end{array}$ & $\begin{array}{l}h_{R}=0.1446 \mathrm{~m} \\
u_{R}=0.0 \mathrm{~m} \mathrm{~s}^{-1}\end{array}$ \\
\hline Test (6) & $\begin{array}{l}\text { Case 2.3: 1-rarefaction 2-shock } \\
\text { (Dam-break type problem, "wet bed") }\end{array}$ & $\begin{aligned} h_{L} & =0.1446 \mathrm{~m} \\
u_{L} & =0.0 \mathrm{~m} \mathrm{~s}^{-1}\end{aligned}$ & $\begin{array}{l}h_{R}=0.0046 \mathrm{~m} \\
u_{R}=0.0 \mathrm{~m} \mathrm{~s}^{-1}\end{array}$ \\
\hline Test (7) & Case 2.4: 1-rarefaction 2-rarefaction & $\begin{array}{l}h_{L}=0.1446 \mathrm{~m} \\
u_{L}=-1.0 \mathrm{~m} \mathrm{~s}^{-1}\end{array}$ & $\begin{array}{l}h_{R}=0.1446 \mathrm{~m} \\
u_{R}=1.0 \mathrm{~m} \mathrm{~s}^{-1}\end{array}$ \\
\hline
\end{tabular}

Table I. Definition of the Riemann problems used in the different tests.
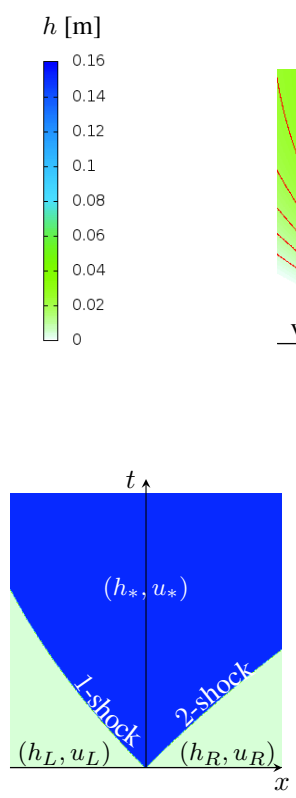

(d) Test (4) - Case 2.1

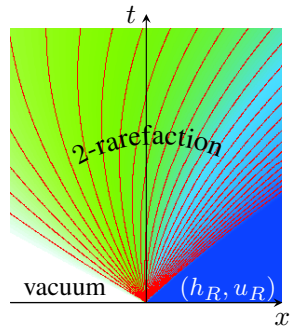

(a) Test (1) - Case 1.1

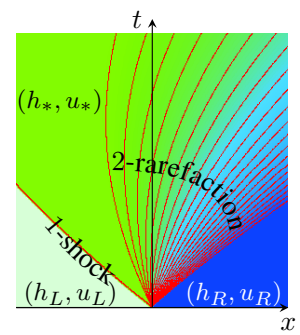

(e) Test (5) - Case 2.2

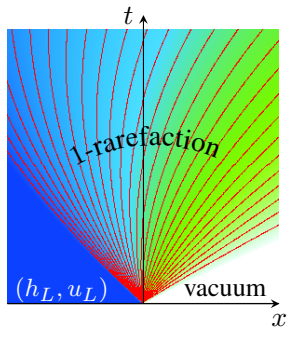

(b) Test (2) - Case 1.2

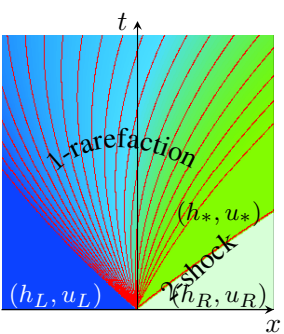

(f) Test (6) - Case 2.3

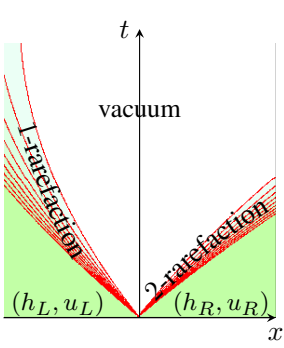

(c) Test (3) - Case 1.3

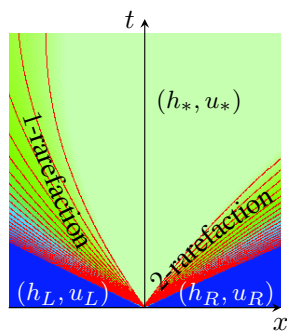

(g) Test (7) - Case 2.4

Figure 5. Projection of the function $h(x, t)$ on the plane $(x, t)$ illustrating the wave patterns. The color scale represents the thickness of the granular mass. Note that the waves have a parabolic shape, contrary to the classic shallow-water system where the waves are straight lines. 
Test (1): $h(x, t)$

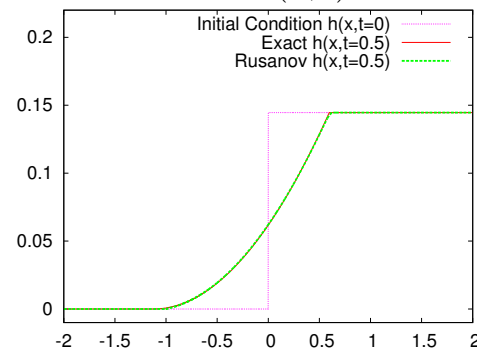

Test (4): $h(x, t)$

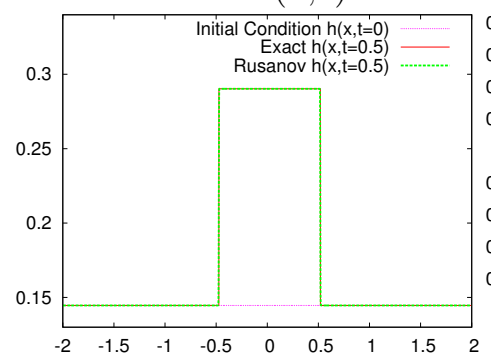

Test (7): $h(x, t)$

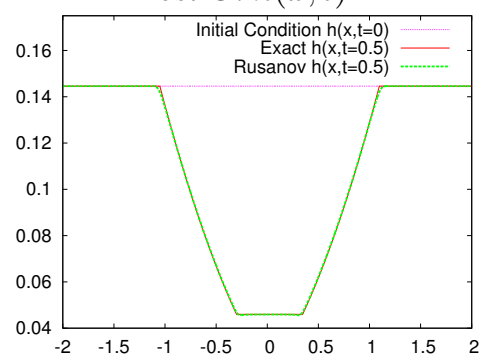

Test (2): $u(x, t)$

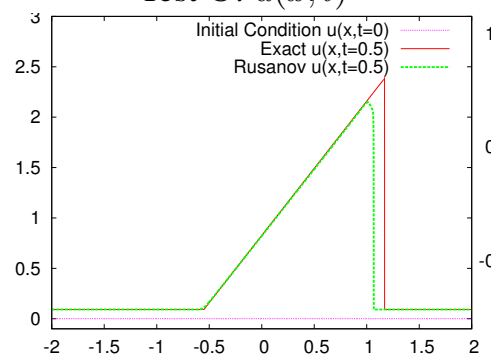

Test (5): $u(x, t)$

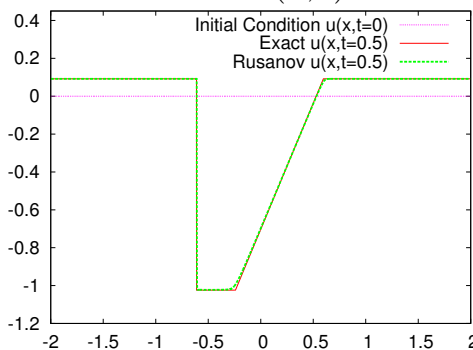

Test (2): $h(x, t)$

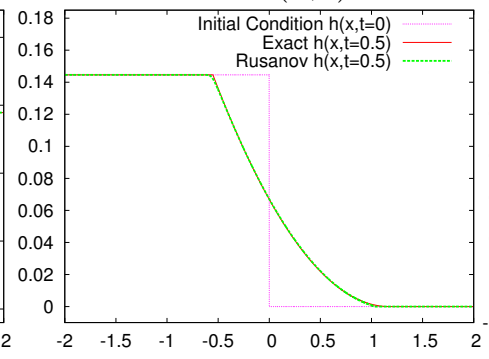

Test (5): $h(x, t)$
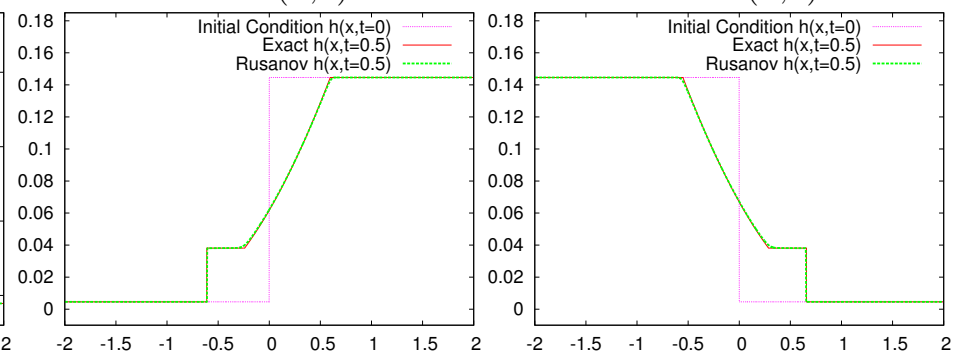

Test (3): $u(x, t)$

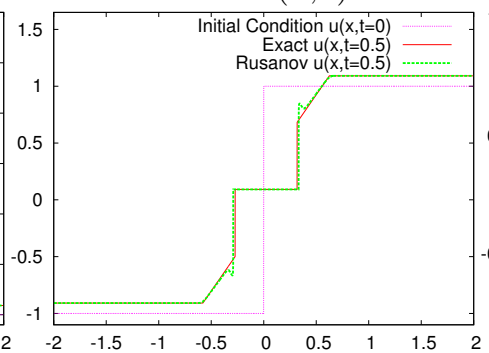

Test (6): $u(x, t)$

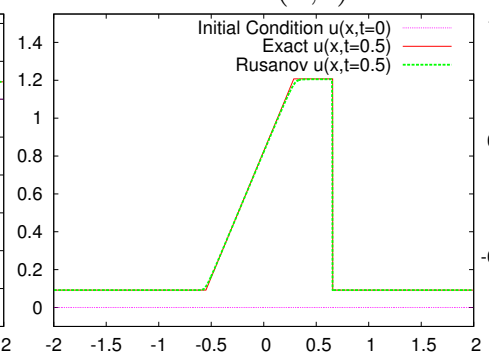

Test (1): $u(x, t)$

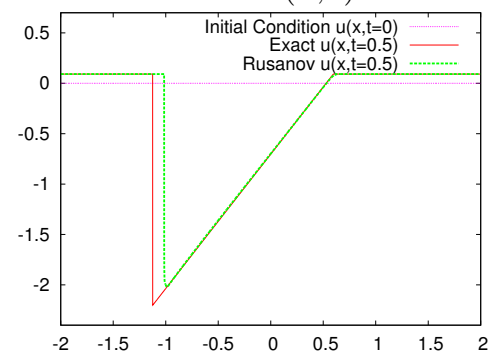

Test (3): $h(x, t)$

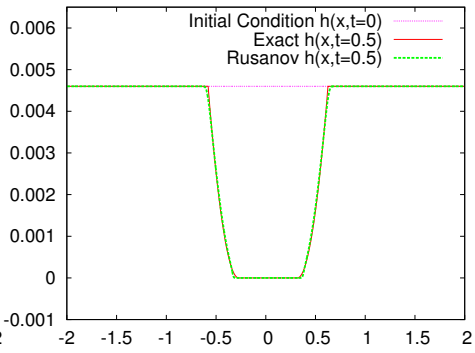

Test (6): $h(x, t)$

Test (4): $u(x, t)$

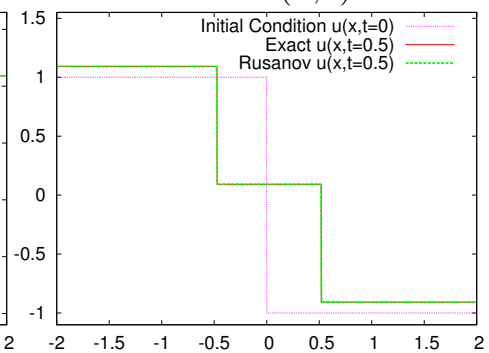

Test (7): $u(x, t)$

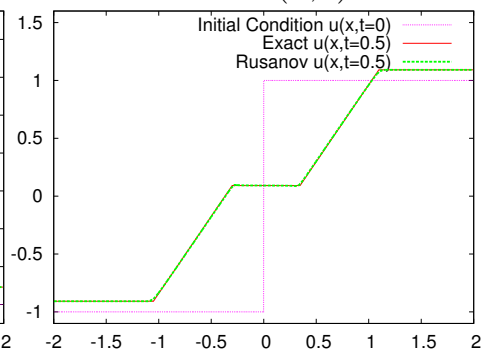

Figure 6. Comparison of the exact solution with the numerical solution computed with the Rusanov scheme (flow-depth on the top and depth-averaged flow velocity on the bottom) at $t=0 \mathrm{~s}$ and $t=0.5 \mathrm{~s}$ for tests (1)-(7) of Table I. The numerical grid is 5000 cells with $\mathrm{CFL}=0.5, m \simeq 0.1839 \mathrm{~m} \mathrm{~s}^{-2}$ and $g_{z} \simeq 9.0957 \mathrm{~m} \mathrm{~s}^{-2}$. 


\section{A SPECIFIC CASE: THE "DAM-BREAK” PROBLEM}

The problem of a dam breaking suddenly is a paradigm for the shallow water model. For our system of equations, the analogous problem is when an infinite mass of granular material is suddenly released from rest over an inclined plane.

First case: "dry bed". Here, a granular material flows over a rough plane inclined at an angle $\varphi$. We assume that a granular mass of constant depth $h_{L}>0$ at $x<0$, initially at rest, is suddenly released at $t=0 \mathrm{~s}$ :

$$
(h, h u)(x, 0)= \begin{cases}\left(h_{L}, 0\right) & \text { if } x<0 \\ (0,0) & \text { if } x>0 .\end{cases}
$$

Using the exact solution presented in section 3 (case 1.2), the spatio-temporal change of the flowdepth is sketched in Figure $7 \mathrm{a}$ and described by

$$
h(x, t)= \begin{cases}h_{L}, & \text { if } x \leq\left(\frac{m t}{2}-c_{L}\right) t, \\ \frac{1}{9 g_{z}}\left(2 c_{L}-\frac{x}{t}+\frac{m t}{2}\right)^{2}, & \text { if }\left(\frac{m t}{2}-c_{L}\right) t<x \leq\left(2 c_{L}+\frac{m t}{2}\right) t, \\ 0, & \text { if } x>\left(2 c_{L}+\frac{m t}{2}\right) t,\end{cases}
$$

and the spatio-temporal change of the depth-averaged flow velocity is described by

$$
u(x, t)= \begin{cases}m t, & \text { if } x \leq\left(\frac{m t}{2}-c_{L}\right) t \\ \frac{2}{3}\left(c_{L}+\frac{x}{t}+m t\right), & \text { if }\left(\frac{m t}{2}-c_{L}\right) t<x \leq\left(2 c_{L}+\frac{m t}{2}\right) t .\end{cases}
$$

The regions $x<\left(\frac{m t}{2}-c_{L}\right) t$ and $x>\left(2 c_{L}+\frac{m t}{2}\right) t$ are the spatio-temporal zones where the thickness is not affected by the dam removal. We obtain the solution computed by Mangeney et al. [28].

Second case: "wet bed". Here we consider the case where a granular mass is initially released from rest over an inclined plane of slope angle $\varphi$, covered by a layer made of the same material. This case may mimic erosion processes of natural flows traveling over deposits built up by earlier events (Mangeney et al. [29]). It is an obvious extension to the removal of a dam between two reservoirs containing material at different heights and corresponds to the Riemann problem

$$
(h, h u)(x, 0)= \begin{cases}\left(h_{L}, 0\right) & \text { if } x<0, \\ \left(h_{R}, 0\right) & \text { if } x>0,\end{cases}
$$

with $h_{L}>h_{R}>0$. Using the solution presented in section 3 (case 2.3), the spatio-temporal change in the flow-depth is sketched in Figure $7 \mathrm{~b}$ and described by

$$
h(x, t)= \begin{cases}h_{L}, & \text { if } x \leq\left(\frac{m t}{2}-c_{L}\right) t, \\ \frac{1}{9 g_{z}}\left(2 c_{L}-\frac{x}{t}+\frac{m t}{2}\right)^{2}, & \text { if }\left(\frac{m t}{2}-c_{L}\right) t<x \leq\left(2 c_{L}-3 c_{*}+\frac{m t}{2}\right) t, \\ h_{*}, & \text { if }\left(2 c_{L}-3 c_{*}+\frac{m t}{2}\right) t<x<\left(\dot{s}+\frac{m t}{2}\right) t, \\ h_{R}, & \text { if } x>\left(\dot{s}+\frac{m t}{2}\right) t,\end{cases}
$$

and the spatio-temporal change in the depth-averaged flow velocity is described by

$$
u(x, t)= \begin{cases}m t, & \text { if } x \leq\left(\frac{m t}{2}-c_{L}\right) t \\ \frac{2}{3}\left(c_{L}+\frac{x}{t}+m t\right), & \text { if }\left(\frac{m t}{2}-c_{L}\right) t<x \leq\left(2 c_{L}-3 c_{*}+\frac{m t}{2}\right) t, \\ 2\left(c_{L}-c_{*}\right)+m t, & \text { if }\left(2 c_{L}-3 c_{*}+\frac{m t}{2}\right) t<x<\left(\dot{s}+\frac{m t}{2}\right) t, \\ m t, & \text { if } x>\left(\dot{s}+\frac{m t}{2}\right) t\end{cases}
$$




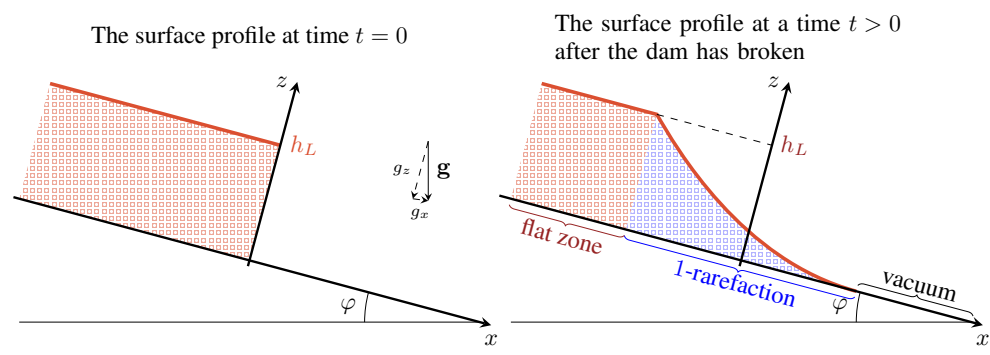

(a) Flow over a dry sloping plane with "dry bed" initial condition.

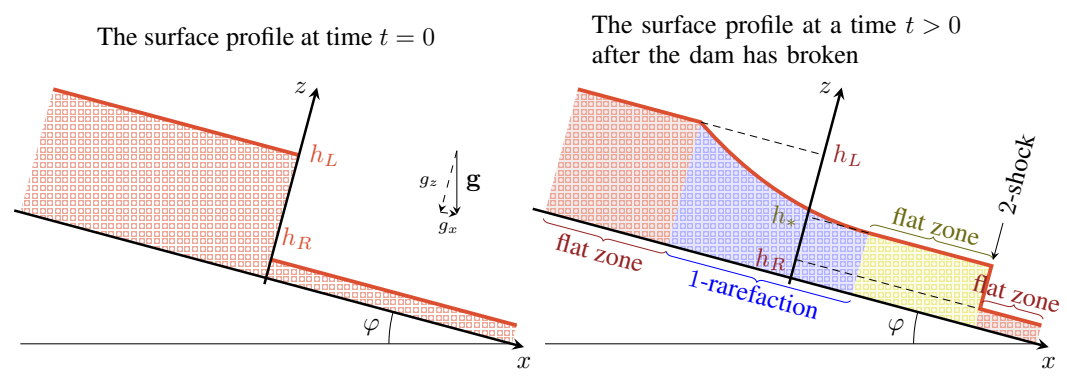

(b) Flow over a sloping plane covered by an erodible bed with "wet bed" initial condition.

Figure 7. Surface profile for the "dam-break" problem: an infinite granular mass is suddenly released from rest over an inclined rough bed (a) and bed covered by a layer made of the same material as the released mass (b).

\begin{tabular}{lcccc}
\hline & Case A: $\delta=0^{\circ}$ & Case B: $\delta=15^{\circ}$ & Case C: $\delta=29^{\circ}$ & $\begin{array}{c}\text { Case D: } \delta=30^{\circ} \\
m=0 \mathrm{~m} \mathrm{~s}^{-2}\end{array}$ \\
\hline$h_{R}=0 \mathrm{~m} / 0 \mathrm{~m}$ & $943 \mathrm{~m} / 0 \mathrm{~m}$ & $687 \mathrm{~m} / 0 \mathrm{~m}$ & $413 \mathrm{~m} / 0 \mathrm{~m}$ & $391 \mathrm{~m} / 0 \mathrm{~m}$ \\
$h_{R}=1 \% h_{L}$ & $795 \mathrm{~m} / 3.423 \mathrm{~m}$ & $539 \mathrm{~m} / 3.423 \mathrm{~m}$ & $267 \mathrm{~m} / 3.423 \mathrm{~m}$ & $243 \mathrm{~m} / 3.423 \mathrm{~m}$ \\
$h_{R}=33.61 \% h_{L}$ & $735 \mathrm{~m} / 12.363 \mathrm{~m}$ & $479 \mathrm{~m} / 12.363 \mathrm{~m}$ & $205 \mathrm{~m} / 12.363 \mathrm{~m}$ & $183 \mathrm{~m} / 12.363 \mathrm{~m}$ \\
$h_{R}=60 \% h_{L}$ & $738 \mathrm{~m} / 15.73 \mathrm{~m}$ & $482 \mathrm{~m} / 15.73 \mathrm{~m}$ & $208 \mathrm{~m} / 15.73 \mathrm{~m}$ & $186 \mathrm{~m} / 15.73 \mathrm{~m}$ \\
$h_{R}=99 \% h_{L}$ & $747 \mathrm{~m} / 19.9 \mathrm{~m}$ & $491 \mathrm{~m} / 19.9 \mathrm{~m}$ & $217 \mathrm{~m} / 19.9 \mathrm{~m}$ & $195 \mathrm{~m} / 19.9 \mathrm{~m}$ \\
\hline
\end{tabular}

Table II. Front position $x_{\text {front }} /$ thickness $h_{*}$ of the intermediate zone obtained with the exact solution for the dam-break problem over a plane with slope angle $\varphi=30^{\circ}$ for different values of $h_{R}$ and different values of the friction angle $\delta$. The position is computed at time $t=15 \mathrm{~s}$ with $h_{L}=20 \mathrm{~m}$.

\subsection{Influence of the friction coefficient on the front position and velocity}

To illustrate the behavior of the exact solution in the dam-break problems, fluid heights are calculated for the case of the instantaneous release of an infinitely-long $h_{L}=20 \mathrm{~m}$ high granular mass on an inclined bottom $\left(\varphi=30^{\circ}\right)$ without friction $\left(\delta=0^{\circ}\right)$, with $\delta=15^{\circ}$, with $\delta=29^{\circ}$ and with $\delta=30^{\circ}$, hereafter referred to as case $\mathrm{A}$, case $\mathrm{B}$, case $\mathrm{C}$ and case $\mathrm{D}$, respectively. The solutions are compared at time $t=15 \mathrm{~s}$ for different values of $h_{R}$ (Table II). As expected, as the friction increases, the front travels over a smaller distance whatever $h_{R}$. The intermediate state $h_{*}$ and the length of the region where $h=h_{*}$ is not affected by the value of the friction coefficient. On the contrary, the value of $h_{R}$ strongly changes $h_{*}$ and the length of the zone where $h=h_{*}$ : both quantities increase with $h_{R}$.

To obtain better insight into the influence of the friction coefficient and of the ratio $h_{R} / h_{L}$ on the front behavior, let us look at the expression for the front position $x_{\text {front }}$ as a function of the time $t$ :

$$
x_{\text {front }}(t)= \begin{cases}\left(2 \sqrt{g_{z} h_{L}}+\frac{m t}{2}\right) t, & \text { if } h_{R}=0, \\ \left(\dot{s}+\frac{m t}{2}\right) t, & \text { if } 0<h_{R}<h_{L} .\end{cases}
$$




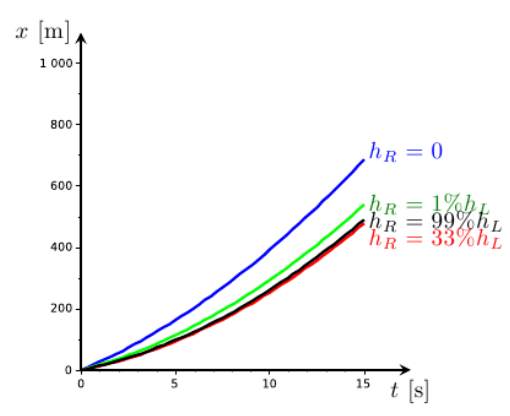

(a) $\delta=15^{\circ}$, thus $m=2.629 \mathrm{~m} \mathrm{~s}^{-2}$

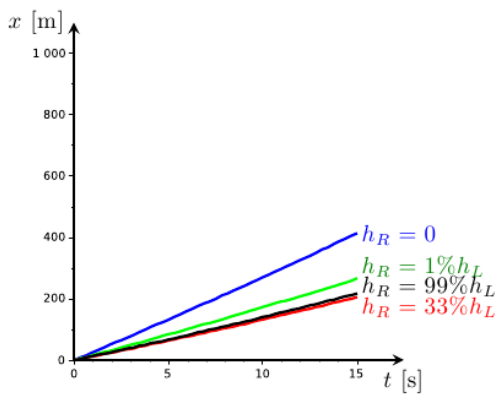

(b) $\delta=29^{\circ}$, thus $m=0.196 \mathrm{~m} \mathrm{~s}^{-2}$

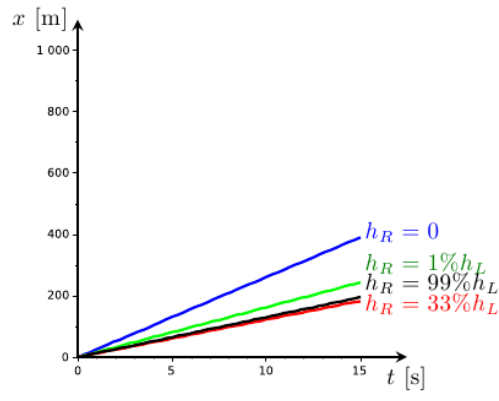

(c) $\delta=30^{\circ}$, thus $m=0.0 \mathrm{~m} \mathrm{~s}^{-2}$

Figure 8. Position of the front $x_{\text {front }}$ as a function of the time $t$ for different values of the friction angle $\delta$. For all figures, $h_{L}=20 \mathrm{~m}$ and $\varphi=30^{\circ}$. For $m>0$, the function $x_{\text {front }}=x_{\text {front }}(t)$ is a parabola; for $m=0$ we obtain the classic behavior of the shallow water system where this function is a straight line.

For $h_{R}>0$, the speed of the front is $\dot{x}_{\text {front }}=\dot{s}+m t / 2$ where $\dot{s}$ depends only on the two constant initial values $h_{L}$ and $h_{R}$ :

$$
\dot{s}=h_{*} \sqrt{\frac{g_{z}}{2} \frac{h_{*}+h_{R}}{h_{*} h_{R}}},
$$

and the constant value $h_{*} \in\left(h_{R} ; h_{L}\right)$ is the solution of the nonlinear equation

$$
\sqrt{8}\left(\sqrt{h_{L}}-\sqrt{h_{*}}\right)=\left(h_{*}-h_{R}\right) \sqrt{\frac{1}{h_{R}}+\frac{1}{h_{*}}} .
$$

We also know that on both sides of the shock wave the velocity of the flow relative to the shock wave is negative: $\dot{s}>u_{*}>u_{R}=0$. In Figure 8 we draw the position of the front $x_{\text {front }}$ as a function of time for different values of the friction angle $\delta$ and for different values of the ratio thickness $h_{R} / h_{L}$ with $h_{L}=20 \mathrm{~m}$ and $\varphi=30^{\circ}$. For $m \neq 0$ the function $x_{\text {front }}=x_{\text {front }}(t)$ has a parabola shape whereas for $m=0$ we obtain the classic straight line of the shallow water system. As expected, the front always accelerates for friction angle $\delta$ smaller than $30^{\circ}$. When $\delta=30^{\circ}(m=0)$, i.e. when the friction compensates for gravity, the front immediately reaches a steady velocity, as in the classical Saint-Venant system.

\subsection{Influence of the thickness of the erodible bed on the front position and velocity}

Figure 8 shows that whatever the friction coefficient (i.e. whatever $m$ ) the front travels over a smaller distance when the thickness of the bed layer on the right $h_{R}$ increases towards a value $h_{R} \simeq 33.61 \% h_{L}$. Then the front position increases slightly with $h_{R}$. This critical thickness $h_{R}^{c} \simeq$ $33.61 \% h_{L}$ is independent of the friction angle $\delta$ for a given $h_{L}$. Note that a strong decrease of the front position for very small $h_{R}$ (about $1 \% h_{L}$ ) is observed, whereas for $h_{R}>h_{R}^{c}$, the front position (and velocity) is almost insensitive to the value of $h_{R}$.

Let us look in more detail at the influence of the thickness of the erodible bed by focusing on the case of the instantaneous release of an infinitely-long granular mass of thickness $h_{L}>0$ on an inclined bottom covered or not by a layer made of the same granular material. To compute the position of the front wave as a function of the ratio $\xi \equiv h_{R} / h_{L}$, we introduce the normalized speed of the front relative to the accelerated frame:

$$
\psi \equiv \frac{\frac{x_{\text {front }}(t)}{t}-\frac{m t}{2}}{\sqrt{g_{z} h_{L}}} .
$$

If $\xi=0$ (i.e. $h_{R}=0$ ) the front of the flow is the tail of the rarefaction wave, whereas, if $\xi \in(0 ; 1)$ (i.e. $h_{L}>h_{R}>0$ ), the front of the flow is located at the shock wave, thus

$$
\psi(\xi)= \begin{cases}2, & \text { if } \xi=0, \\ \frac{\dot{s}}{\sqrt{g_{z} h_{L}}}=\frac{\eta(\xi)}{\sqrt{2}} \sqrt{\frac{1}{\xi}+\frac{1}{\eta(\xi)}}, & \text { if } 0<\xi<1,\end{cases}
$$




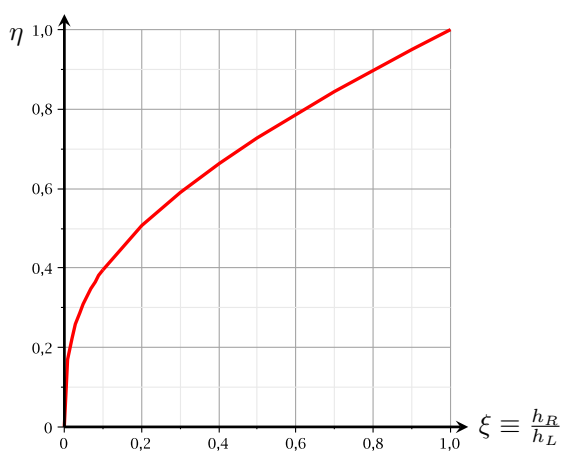

(a) Normalized height $\eta(\xi)=h_{*} / h_{L}$ of the intermediate flat zone.

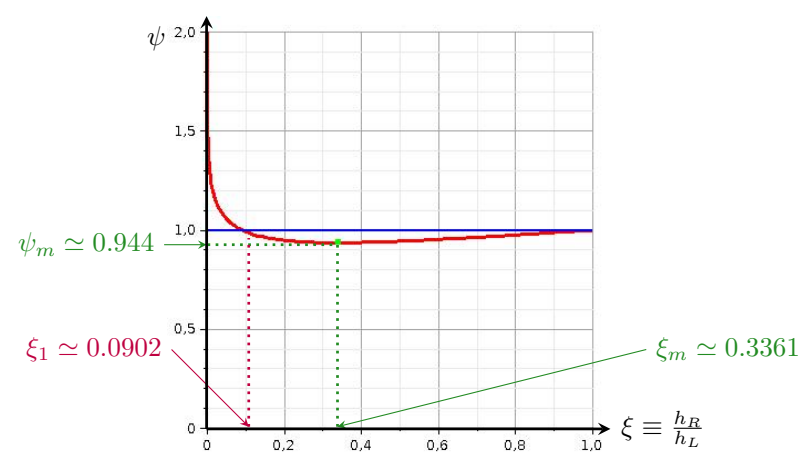

(b) Normalized speed of the shock at the front of the flow.

Figure 9. Normalized characteristics of the flow as a function of the normalized thickness of the erodible layer.

where $\eta=\eta(\xi) \equiv \frac{h_{*}}{h_{L}} \in(\xi ; 1)$ is implicitly defined by the following nonlinear equation

$$
\sqrt{8}(1-\sqrt{\eta})=(\eta-\xi) \sqrt{\frac{1}{\xi}+\frac{1}{\eta}}
$$

Figure 9a shows the ratio $\eta$ as a function of the ratio $\xi$ : as expected, $\eta(\xi) \underset{\xi \rightarrow 1}{\longrightarrow} 1, \eta(\xi) \underset{\xi \rightarrow 0}{\longrightarrow} 0$ and $\eta^{\prime}(\xi)>0$. Figure $9 \mathrm{~b}$ represents the normalized speed of the front $\psi$ as a function of the ratio $\xi$ : $\psi(\xi) \underset{\xi \rightarrow 1}{\longrightarrow} 1$ and $\psi(\xi)=1$ for $\xi=\xi_{1} \simeq 0.0902$. We observe non-monotonous behavior: denoting $\xi_{m}$ the value such that $\psi^{\prime}\left(\xi_{m}\right)=0$, then for $\xi<\xi_{m}, \psi$ decreases as $\xi$ increases and for $\xi>\xi_{m}, \psi$ increases with $\xi$. To estimate $\xi_{m}$ we observe that

$$
\psi^{\prime}(\xi)=\frac{\eta^{2}(\xi)+2 \eta(\xi) \xi+\xi^{2}-\sqrt{\eta^{5}(\xi)} \sqrt{\frac{2}{\xi}+\frac{2}{\eta(\xi)}}}{\xi \sqrt{\frac{2}{\xi}+\frac{2}{\eta(\xi)}}\left(\xi \sqrt{\eta^{3}(\xi)} \sqrt{\frac{8}{\xi}+\frac{8}{\eta(\xi)}}+\xi \eta(\xi)+2 \eta^{2}(\xi)+\xi^{2}\right)} .
$$

Thus, solving $\psi^{\prime}(\xi)=0$ numerically together with the equation (4), we obtain $\xi_{m} \simeq 0.3361$ and $\psi\left(\xi_{m}\right) \simeq 0.944$, so that a minimum velocity $\left(0.944 \sqrt{g_{z} h_{L}}\right)$ of the front relative to the accelerated reference frame is obtained for $h_{R} \simeq 33.61 \% h_{L}$. We conclude that

$$
0.944 \sqrt{g_{z} h_{L}} \leq \dot{s} \leq 2 \sqrt{g_{z} h_{L}} .
$$

\subsection{Discussion on the physical meaning of the solution.}

A great amount of experimental and numerical work has been done on granular flows over rough or erodible inclined beds (e.g. Gray and Ancey [11], Mallogi et al. [25], Mangeney et al. [27, 29], Mangeney-Castelnau et al. [32], Pouliquen and Forterre [37], Roche et al. [38]). Even though it is quite difficult to compare these results to our very simplified exact solution, let us discuss the general trends and the rough order of magnitudes of the solution with respect to laboratory experiments in some specific cases.

For granular collapse over rough beds with slope angles smaller than the repose angle of the granular mass involved (hereafter called moderate slope), the exact solution presented here reproduces the mean dynamics and deposition of granular collapse over horizontal or inclined beds (see Mangeney et al. [29], Mangeney-Castelnau et al. [32]). On the contrary, for granular collapse over an erodible bed of moderate slope, the solution proposed here does not agree with experimental observations. In particular, contrary to what we obtained here using the exact solution 
(Figure 11b), experimental results show that the front velocity increases with the thickness of the erodible bed, i.e. with $\xi$ (see Mangeney et al. [29]). Numerical simulation by Farin et al. [7] shows indeed that when using a Coulomb-like friction law, the front position and velocity decrease with increasing $h_{R}$. However, it is possible to reproduce the increase in front position and velocity observed experimentally when using the Pouliquen and Forterre friction law in which the friction coefficient depends on both the velocity and thickness of the flow (Farin et al. [7]).

Even though the analogy between the exact solution and the experimental studies on granular flows over erodible beds is very difficult due to the simplified solution developed here, it seems that the thin layer model with a constant Coulomb friction law and its associated solution lacks a strong physical basis to quantitatively reproduce the experimental observations. Indeed, the description of erosion/deposition processes is still an open issue due to the lack of precise understanding and quantification of the mechanisms and parameters that control the static/flowing transition in granular media (e.g. Bouchut et al. [2], Deboeuf et al. [5], Gray and Ancey [11], Mangeney et al. [27, 31]).

\section{CONCLUSION}

The key results of this paper are the complete general exact solution to the Riemann problem for the shallow water equations with a Mohr-Coulomb type friction law and the mathematical proof of the solution derivation. The solution comprises two waves, a 1-wave and a 2-wave, separated by an intermediate constant state. Each of these two waves may only be a rarefaction or a shock wave. Thus, there can only be 7 possible combinations of waves. All these combinations, which constitute the complete set of solutions to the Riemann problem, have been derived and discussed here. We have proposed a method making it possible to find the intermediate state by solving a nonlinear equation. The results computed with this method have been compared successfully with the numerical solutions of some Riemann problems calculated by means of the Rusanov scheme, a Godunov-type approximate Riemann solver.

We have also investigated the influence of the thickness of the erodible bed on the front speed for the dam-break problem. When compared to existing experimental results, the exact solution seems to reasonably well reproduce granular flows over a rough bed. On the other hand, the qualitative and quantitative results obtained for granular flows over erodible layers are very different from the observations, suggesting that more sophisticated flow laws should be used. In particular, the existence of static and flowing zones within the granular mass observed when a mass is flowing over an erodible bed or segregation and recirculation processes must be taken into account (see e.g. Gray and Ancey [11], Mangeney et al. [27, 29, 31]). Force chains in granular materials may also play a role in erosion/deposition processes, as observed at the onset of destabilization in granular layers, preventing the use of continuum models for accurate description of these processes (e.g. Deboeuf et al. [5]).

The detailed derivation and presentation of the solution is mostly dedicated to geophysicists, physicists and mathematicians for direct use or for possible generalization to more complicated geometries or flow laws. These solutions can be extended to go beyond the specific problem of granular flows because shallow water equations are widely used in other fields such as lava, glacier and river flows, oceanography and atmospheric studies.

\section{A. PROOF OF THE EXACT SOLUTION OF THE RIEMANN PROBLEM}

In this section, we solve the Riemann problem for system (1) using classical theory of hyperbolic problems. For $x \in \mathbb{R}$ and $t>0$, by introducing the velocity $v(x, t) \equiv u(x, t)-m t$, the nonlinear hyperbolic system (1) can be written concisely as

$$
\partial_{t} \mathbf{V}+\partial_{x} \mathbf{F}(\mathbf{V})=\mathbf{0} \quad \text { where } \quad \mathbf{V} \equiv\left(\begin{array}{c}
h \\
h v
\end{array}\right) \quad \text { and } \quad \mathbf{F}(\mathbf{V}) \equiv\left(\begin{array}{c}
h(v+m t) \\
h v(v+m t)+\frac{g_{z}}{2} h^{2}
\end{array}\right) .
$$


Let us consider the Riemann problem, i.e. the initial condition

$$
\mathbf{V}(x, t=0)= \begin{cases}\mathbf{V}_{L} & \text { if } x<0, \\ \mathbf{V}_{R} & \text { if } x>0\end{cases}
$$

where $\mathbf{V}_{L} \equiv\left(h_{L},(h v)_{L}\right)$ and $\mathbf{V}_{R} \equiv\left(h_{R},(h v)_{R}\right)$ are two constant states.

First, we describe here the mathematical properties of the system (5). We follow with a detailed description of the construction of the analytical solution following four steps: (i) the eigenstructure of the system; (ii) the solution for rarefaction waves; (iii) the solution for shock waves, (iv) the solution for the general Riemann problem. Finally, we verify that the total energy function is an entropy function for the system.

\section{A.1. Hyperbolicity}

To perform the hyperbolicity analysis, we rewrite (5) in the quasilinear following form

$$
\partial_{t} \mathbf{W}+\mathbb{A}(\mathbf{W}) \partial_{x} \mathbf{W}=\mathbf{0} \quad \text { where } \quad \mathbf{W} \equiv\left(\begin{array}{l}
h \\
v
\end{array}\right) \quad \text { and } \quad \mathbb{A}(\mathbf{W}) \equiv\left(\begin{array}{cc}
v+m t & h \\
g z & v+m t
\end{array}\right) .
$$

The matrix $\mathbb{A}(\mathbf{W})$ has the following two real eigenvalues (characteristic speeds), written in increasing order,

$$
\lambda_{1}(\mathbf{W})=v+m t-\sqrt{g_{z} h} \leq \lambda_{2}(\mathbf{W})=v+m t+\sqrt{g_{z} h} .
$$

If $h>0$, the eigenvalues are distinct thus the system (5) is strictly hyperbolic.

\section{A.2. Wave structure}

We seek a solution of the form $\mathbf{V}(x, t)=\mathbf{V}(\zeta)$, i.e. a self-similar solution in the variable $\zeta$, meaning that the solutions at different times "can be obtained from one another by a similarity transformation". This means that the solution is constant along any ray $\zeta$ in the $(x, t)$-plane. It can be shown that self-similar solutions of the Riemann problem are composites of constant states, shocks joining constant states, and rarefaction waves connecting constant states or contact discontinuities. More precisely, since for a strictly hyperbolic system waves of different families are strictly separated, any self-similar solution to the Riemann problem for a $n \times n$ system comprises $n+1$ constant states $\mathbf{V}_{k}, k=1, \ldots, n+1$. States $\mathbf{V}_{k-1}$ and $\mathbf{V}_{k}$ are joined by a wave of the $k$ family which, in general, may consist of $k$-rarefactions, $k$-shocks and/or $k$-contact discontinuities.

In our case $n=2$ so that the exact solution is composed of three constant states, $\mathbf{V}_{L}, \mathbf{V}_{*}$ and $\mathbf{V}_{R}$, separated by two waves. Next, the admissible wave structure for our system is described, and the complete set of solutions to the Riemann problem is given. We describe in detail how to compute the intermediate constant state $\mathbf{V}_{*}$ and the waves separating these three constant states and we will see that the similarity variable $\zeta$ is $\zeta \equiv x / t-m t / 2$.

A.2.1. Eigenstructure and Riemann Invariants. The right eigenvectors associated with the characteristic speeds are

$$
\mathbf{r}_{1}(\mathbf{W})=\left(-\frac{2 \sqrt{h}}{3 \sqrt{g_{z}}}, \frac{2}{3}\right)^{T}, \quad \quad \mathbf{r}_{2}(\mathbf{W})=\left(\frac{2 \sqrt{h}}{3 \sqrt{g_{z}}}, \frac{2}{3}\right)^{T} .
$$

It is immediately verified that $\left(\nabla \lambda_{k}(\mathbf{W})\right)^{T} \cdot \mathbf{r}_{k}(\mathbf{W})=1$ for $k=1,2$ and for any $\mathbf{W}$ in the domain of definition of the variables $h$ and $v$, so that characteristic fields are genuinely nonlinear. The genuinely nonlinear character of the eigenvalues implies that all waves are rarefaction or shock waves. Let us define $I_{k}$ as the Riemann invariant of the $k$-field, i.e. $\left(\nabla I_{k}(\mathbf{W})\right)^{T} \cdot \mathbf{r}_{k}(\mathbf{W})=0$, for $k=1,2$. We can choice $I_{1}=v+m t+2 \sqrt{g_{z} h}$ and $I_{2}=v+m t-2 \sqrt{g_{z} h}$. 
A.2.2. Rarefaction waves. A rarefaction wave is a one-parameter family of states connecting a given state and satisfying the differential equations (5). This kind of continuous solution is obtained by determining the so-called integral curves of each genuinely nonlinear mode of the system, that is the curves tangent at any point to the vector field of the associated eigenvector.

1-rarefaction. The condition $\lambda_{1}\left(\mathbf{W}_{L}\right)<\lambda_{1}\left(\mathbf{W}_{*}\right)$ gives $v_{L}+m t-\sqrt{g_{z} h_{L}}<v_{*}+m t-\sqrt{g_{z} h_{*}}$ then $v_{*}>v_{L}+\sqrt{g_{z}}\left(\sqrt{h_{*}}-\sqrt{h_{L}}\right)$. In a $k$-rarefaction wave, the $k$-Riemann invariants are conserved; here $I_{1}\left(\mathbf{W}_{L}\right)=I_{1}\left(\mathbf{W}_{*}\right)$, i.e. $v_{L}+2 \sqrt{g_{z} h_{L}}=v_{*}+2 \sqrt{g_{z} h_{*}}$, then $h_{*} \mapsto v_{*}=v_{L}+$ $2 \sqrt{g_{z}}\left(\sqrt{h_{L}}-\sqrt{h_{*}}\right)$, thus $h_{*}<h_{L}$ and $v_{*}>v_{L}$. We conclude that the states $\mathbf{W}_{*}=\left(h_{*}, v_{*}\right)$ which can be connected to a given left state $\mathbf{W}_{L}=\left(h_{L}, v_{L}\right)$ by a 1-rarefaction wave satisfy the relation

$$
v_{*}=v_{L}+2 \sqrt{g_{z}}\left(\sqrt{h_{L}}-\sqrt{h_{*}}\right) \quad \text { for } h_{*}<h_{L}
$$

and for $h_{*}<h_{L}$ we have $v_{*}^{\prime}\left(h_{*}\right)=-\sqrt{g_{z} / h_{*}}<0$ and $v_{*}^{\prime \prime}\left(h_{*}\right)=\sqrt{g_{z} /\left(4 h_{*}^{3}\right)}>0$ (see Figure 10a). The speed of the 1-rarefaction wave satisfies $\lambda_{1}\left(\mathbf{W}_{L}\right) \leq \frac{\mathrm{d} x}{\mathrm{~d} t} \leq \lambda_{1}\left(\mathbf{W}_{*}\right)$, i.e. $v_{L}+m t / 2-\sqrt{g_{z} h_{L}} \leq x / t \leq v_{*}+m t / 2-\sqrt{g_{z} h_{*}}$, that can be rewritten as

$$
\underbrace{\left(v_{L}+m t-\sqrt{g_{z} h_{L}}\right)}_{\lambda_{1}\left(\mathbf{W}_{L}\right)} t \leq x+\frac{m t^{2}}{2} \leq \underbrace{\left(v_{*}+m t-\sqrt{g_{z} h_{*}}\right)}_{\lambda_{1}\left(\mathbf{W}_{*}\right)} t .
$$

To compute the solution at a point $(\hat{x}, \hat{t})$ at the interior of the wave, we consider the characteristic that connect this point at the origin of the wave $(0,0)$. The characteristic speed verify $\frac{\mathrm{d} x}{\mathrm{~d} t}=$ $\lambda_{1}(\mathbf{W})$, i.e. $\frac{\hat{x}}{\hat{t}}=v+\frac{m \hat{t}}{2}-\sqrt{g_{z} h}$. Moreover, the Riemann invariant is conserved in the wave, thus $v+2 \sqrt{g_{z} h}=v_{L}+2 \sqrt{g_{z} h_{L}}$. We obtain

$$
v(\hat{x}, \hat{t})=\frac{1}{3}\left(v_{L}+2 \sqrt{g_{z} h_{L}}+2 \frac{\hat{x}}{\hat{t}}-m \hat{t}\right), \quad h(\hat{x}, \hat{t})=\frac{1}{9 g_{z}}\left(v_{L}+2 \sqrt{g_{z} h_{L}}-\frac{\hat{x}}{\hat{t}}+\frac{m \hat{t}}{2}\right)^{2} .
$$

2-rarefaction. In the same way, the condition $\lambda_{2}\left(\mathbf{W}_{*}\right)<\lambda_{2}\left(\mathbf{W}_{R}\right)$ gives $v_{*}<v_{R}+$ $\sqrt{g_{z}}\left(\sqrt{h_{R}}-\sqrt{h_{*}}\right)$. In a $k$-rarefaction wave, the $k$-Riemann invariant is conserved; here $I_{2}\left(\mathbf{W}_{*}\right)=I_{2}\left(\mathbf{W}_{R}\right)$, then $h \mapsto v_{*}=v_{R}+2 \sqrt{g_{z}}\left(\sqrt{h_{*}}-\sqrt{h_{R}}\right)$ and thus $h_{*}<h_{R}$ and $v_{*}<v_{R}$. We conclude that the states $\mathbf{W}_{*}=\left(h_{*}, v_{*}\right)$ that can be connected to a given right state $\mathbf{W}_{R}=\left(h_{R}, v_{R}\right)$ by a 2-rarefaction wave verify the relation

$$
v_{*}=v_{R}+2 \sqrt{g_{z}}\left(\sqrt{h_{*}}-\sqrt{h_{R}}\right) \quad \text { for } h_{*}<h_{R}
$$

and for $h_{*}<h_{R}$ we have $v_{*}^{\prime}\left(h_{*}\right)=\sqrt{g_{z} / h_{*}}>0$ and $v_{*}^{\prime \prime}\left(h_{*}\right)=-\sqrt{g_{z} /\left(4 h_{*}^{3}\right)}<0$ (see Figure 10b). The speed of the 2-rarefaction wave satisfies $\lambda_{2}\left(\mathbf{W}_{*}\right) \leq \frac{\mathrm{d} x}{\mathrm{~d} t} \leq \lambda_{2}\left(\mathbf{W}_{R}\right)$, i.e. $v_{*}+m t / 2+\sqrt{g_{z} h_{*}} \leq x / t \leq v_{R}+m t / 2+\sqrt{g_{z} h_{R}}$, that can be rewritten

$$
\underbrace{\left(v_{*}+m t+\sqrt{g_{z} h_{*}}\right)}_{\lambda_{2}\left(\mathbf{W}_{*}\right)} t \leq x+\frac{m t^{2}}{2} \leq \underbrace{\left(v_{R}+m t+\sqrt{g_{z} h_{R}}\right)}_{\lambda_{2}\left(\mathbf{W}_{R}\right)} t .
$$

To compute the solution at a point $(\hat{x}, \hat{t})$ at the interior of the wave, we consider the characteristic that connect this point at the origin of the wave $(0,0)$. The characteristic speed satisfies $\frac{\mathrm{d} x}{\mathrm{~d} t}=\lambda_{2}(\mathbf{W})$, i.e. $\frac{\hat{x}}{\hat{t}}=v+\frac{m \hat{t}}{2}+\sqrt{g_{z} h}$. Moreover, the Riemann invariant is conserved in the wave, thus $v-2 \sqrt{g_{z} h}=v_{R}-2 \sqrt{g h_{R}}$. We obtain

$$
v(\hat{x}, \hat{t})=\frac{1}{3}\left(v_{R}-2 \sqrt{g_{z} h_{R}}+2 \frac{\hat{x}}{\hat{t}}-m \hat{t}\right), \quad h(\hat{x}, \hat{t})=\frac{1}{9 g_{z}}\left(-v_{R}+2 \sqrt{g_{z} h_{R}}+\frac{\hat{x}}{\hat{t}}-\frac{m \hat{t}}{2}\right)^{2} .
$$


A.2.3. Shock waves. Let us turn now to the description of the shock curves. A $k$-shock wave is a piecewise constant discontinuous solution, satisfying the entropy condition, that propagates at a velocity $\dot{\sigma}_{k}$ dependent on the states existing on the two sides of the jump. The conservation variables must respect the Rankine-Hugoniot jump conditions. In the present case, if we note $\left(h_{\ell}, v_{\ell}\right)$ and $\left(h_{r}, v_{r}\right)$ these two states, the jump conditions assume the form

$$
\left\{\begin{array}{l}
\dot{\sigma}_{k}=\frac{h_{\ell}\left(v_{\ell}+m t\right)-h_{r}\left(v_{r}+m t\right)}{h_{\ell}-h_{r}} \\
\dot{\sigma}_{k}=\frac{h_{\ell} v_{\ell}\left(v_{\ell}+m t\right)+\left(g_{z} / 2\right) h_{\ell}^{2}-h_{r} v_{r}\left(v_{r}+m t\right)-\left(g_{z} / 2\right) h_{r}^{2}}{h_{\ell} v_{\ell}-h_{r} v_{r}} .
\end{array}\right.
$$

The curve $x=\sigma_{k}(t)$ is the shock path and $\dot{\sigma}_{k}$ is the shock velocity.

1-shock. We would like to determine all the states $\mathbf{W}_{*}=\left(h_{*}, v_{*}\right)$ that can be connected to a given state $\mathbf{W}_{L}=\left(h_{L}, v_{L}\right)$ by an entropic discontinuity (1-shock) at speed $\dot{\sigma}_{1}$. The discontinuity satisfies the Lax entropy conditions if

$$
\left\{\begin{array}{l}
\lambda_{1}\left(\mathbf{W}_{*}\right)<\dot{\sigma}_{1}<\lambda_{2}\left(\mathbf{W}_{*}\right), \\
\dot{\sigma}_{1}<\lambda_{1}\left(\mathbf{W}_{\mathbf{L}}\right) .
\end{array}\right.
$$

Thus the 1-shock is admissible for $h_{*}>h_{L}$ and $v_{*}<v_{L}$. Eliminating $\dot{\sigma}_{1}$ in Rankine-Hugoniot jump conditions we obtain

$$
h_{*} \mapsto v_{*}=v_{L}+\left(h_{L}-h_{*}\right) \sqrt{\frac{g_{z}}{2} \frac{h_{L}+h_{*}}{h_{L} h_{*}}} \quad \text { for } h_{*}>h_{L}
$$

and the speed of the 1 -shock satisfies $\frac{\mathrm{d} x}{\mathrm{~d} t}=\dot{\sigma}_{1}=v_{L}+m t-h_{*} \sqrt{\frac{g_{z}}{2} \frac{h_{L}+h_{*}}{h_{L} h_{*}}}$, i.e. $\frac{x}{t}=v_{L}+\frac{m t}{2}-h_{*} \sqrt{\frac{g_{z}}{2} \frac{h_{L}+h_{*}}{h_{L} h_{*}}}$, that can be written $x+m t^{2} / 2=\dot{\sigma}_{1} t$. For $h_{*}>h_{L}$, we have $v_{*}^{\prime}\left(h_{*}\right)=-\sqrt{g_{z}}\left(h_{L} h_{*}+2 h_{*}^{2}+h_{L}^{2}\right) / \sqrt{8 h_{*}^{3} h_{L}\left(h_{L}+h_{*}\right)}<0 \quad$ and $\quad v_{*}^{\prime \prime}\left(h_{*}\right)=$ $\sqrt{g_{z} h_{L}^{3}}\left(5 h_{*}+3 h_{L}\right) / \sqrt{32 h^{5}\left(h_{L}+h_{*}\right)^{3}}>0$ (see Figure 10c).

2-shock. In the same way, we would like to determine all the states $\mathbf{W}_{*}=\left(h_{*}, v_{*}\right)$ that can be connected to a given right state $\mathbf{W}_{R}=\left(h_{R}, v_{R}\right)$ by an entropic discontinuity (2-shock) at speed $\dot{\sigma}_{2}$. The discontinuity satisfies the Lax entropy conditions if

$$
\left\{\begin{array}{l}
\lambda_{2}\left(\mathbf{W}_{R}\right)<\dot{\sigma}_{2}, \\
\lambda_{1}\left(\mathbf{W}_{*}\right)<\dot{\sigma}_{2}<\lambda_{2}\left(\mathbf{W}_{*}\right),
\end{array}\right.
$$

which implies $h_{*}>h_{R}$ and $v_{*}>v_{R}$. Eliminating $\dot{\sigma}_{2}$ in the Rankine-Hugoniot jump conditions we obtain

$$
h_{R} \mapsto v_{*}=v_{R}+\left(h_{*}-h_{R}\right) \sqrt{\frac{g_{z}}{2} \frac{h_{*}+h_{R}}{h_{*} h_{R}}} \quad \text { for } h_{*}>h_{R}
$$

and the speed of the 2-shock satisfies $\frac{\mathrm{d} x}{\mathrm{~d} t}=\dot{\sigma}_{2}=v_{R}+m t+h_{*} \sqrt{\frac{g_{z}}{2} \frac{h_{*}+h_{R}}{h_{*} h_{R}}}$, i.e. $\frac{x}{t}=v_{R}+\frac{m t}{2}+h_{*} \sqrt{\frac{g_{z}}{2} \frac{h_{R}+h_{*}}{h_{R} h_{*}}}$, that can be written $x+m t^{2} / 2=\dot{\sigma}_{2} t$. For $h_{*}>h_{R}$, we have $v_{*}^{\prime}\left(h_{*}\right)=\sqrt{g_{z}}\left(h_{*} h_{R}+h_{R}^{2}+2 h_{*}^{2}\right) / \sqrt{8 h_{*}^{3} h_{R}\left(h_{*}+h_{R}\right)}>0 \quad$ and $\quad v_{*}^{\prime \prime}\left(h_{*}\right)=$ $-\sqrt{g_{z} h_{R}^{3}}\left(5 h_{*}+3 h_{R}\right) / \sqrt{32 h_{*}^{5}\left(h_{*}+h_{R}\right)^{3}}<0$ (see Figure 10d).

Remark. It is interesting to note that as $h_{*} \rightarrow h_{L}$ (resp. $h_{*} \rightarrow h_{R}$ ), we find that $\dot{\sigma}_{1} \rightarrow v_{L}-\sqrt{g_{z} h_{L}}$ (resp. $\dot{\sigma}_{2} \rightarrow v_{R}-\sqrt{g_{z} h_{R}}$ ). In other words, the speed of infinitesimal discontinuities is the same as a characteristic speed. 


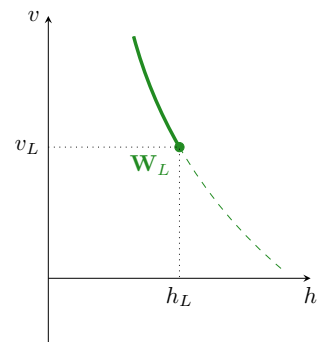

(a) Set of states $\mathbf{W}_{*}$ that can be connected to $\mathbf{W}_{L}$ by a 1-rarefaction wave. The full integral curve is shown as dashed line.

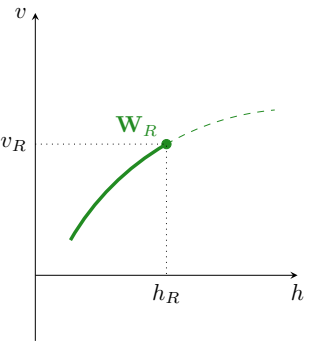

(b) Set of states $\mathbf{W}_{*}$ that can be connected to $\mathbf{W}_{R}$ by a 2-rarefaction wave. The full integral curve is shown as dashed line.

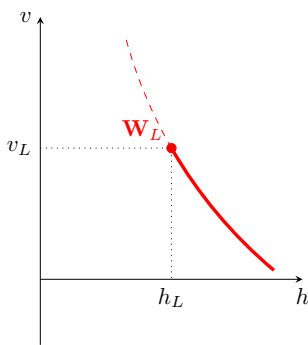

(c) Set of states $\mathbf{W}_{*}$ that can be connected to $\mathbf{W}_{L}$ by a 1 -shock wave satisfying the RankineHugoniot jump conditions and the Lax admissibility conditions. The entropyviolating branch of the Hugoniot locus is shown as dashed line.

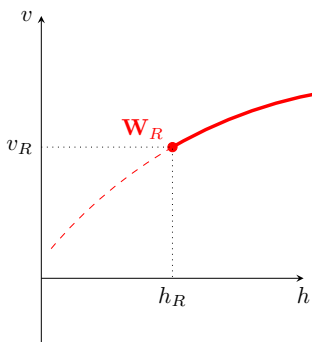

(d) Set of states $\mathbf{W}_{*}$ that can be connected to $\mathbf{W}_{R}$ by a 2 -shock wave satisfying the RankineHugoniot jump conditions and the Lax admissibility conditions. The entropyviolating branch of the Hugoniot locus is shown as dashed line.

Figure 10. Rarefaction and shock waves.

\section{A.3. Complete set of solutions of Riemann Problems.}

Let us now consider the Riemann problem (6) where $h_{L} \geq 0, h_{R} \geq 0, v_{L} \in \mathbb{R}$ and $v_{R} \in \mathbb{R}$ are constant. The solution consist of at most three constant states (including $\left(h_{L},(h v)_{L}\right)$ and $\left.\left(h_{R},(h v)_{R}\right)\right)$ separated by a $k$-rarefaction or a $k$-admissible shock wave, $k=1,2$. So, for a left state $\mathbf{W}_{L}=\left(h_{L}, v_{L}\right)$ and a right state $\mathbf{W}_{R}=\left(h_{R}, v_{R}\right)$, there are only four possible combinations of solutions: we construct a solution composed by a 1-wave and a 2 -wave separated by an intermediate state $\mathbf{W}_{*}=\left(h_{*}, v_{*}\right)$ as in the following figure.

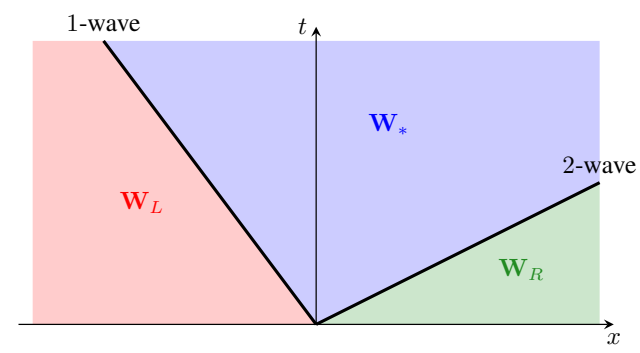

The variables that need to be determined to fully characterize the solution are: the intermediate constant state $\mathbf{W}_{*}$, the speed of shocks $\dot{\sigma}_{1}$ and $\dot{\sigma}_{2}$, the states in rarefaction waves $\mathbf{W}_{1 \text {-rar }}$ and $\mathbf{W}_{2 \text {-rar }}$.

Intermediate state We begin by characterizing the intermediate state $\mathbf{W}_{*}=\left(h_{*}, v_{*}\right)$ that can be connected to the given left state $\mathbf{W}_{L}=\left(h_{L}, v_{L}\right)$ by a 1-curve and to the given right state $\mathbf{W}_{R}=\left(h_{R}, v_{R}\right)$ by a 2 -curve. The intermediate state

- is connected to a left state $\left(h_{L}, v_{L}\right)$ by a 1 -wave family curve that has equation

$$
v_{*}= \begin{cases}v_{L}-\left(h_{*}-h_{L}\right) \frac{2 \sqrt{g_{z}}}{\sqrt{h_{L}}+\sqrt{h_{*}}} & \text { if } h_{*} \leq h_{L}(1 \text {-rarefaction), } \\ v_{L}-\left(h_{*}-h_{L}\right) \sqrt{\frac{g_{z}}{2} \frac{h_{*}+h_{L}}{h_{*} h_{L}}} & \text { if } h_{*}>h_{L}(1 \text {-shock); }\end{cases}
$$

- is connected to a right state $\left(h_{R}, v_{R}\right)$ by a 2 -wave family curve that has equation

$$
v_{*}= \begin{cases}v_{R}+\left(h_{*}-h_{R}\right) \frac{2 \sqrt{g_{z}}}{\sqrt{h_{R}}+\sqrt{h_{*}}} & \text { if } h_{*} \leq h_{R} \text { (2-rarefaction), } \\ v_{R}+\left(h_{*}-h_{R}\right) \sqrt{\frac{g_{z}}{2} \frac{h_{*}+h_{R}}{h_{*} h_{R}}} & \text { if } h_{*}>h_{R} \text { (2-shock). }\end{cases}
$$


Thus, $v_{*}=v_{R}+\left(h_{*}-h_{R}\right) z\left(h_{*}, h_{R}\right)$ and $h_{*}$ is the root of the function

$$
f(h)=v_{R}-v_{L}+\left(h-h_{L}\right) z\left(h, h_{L}\right)+\left(h-h_{R}\right) z\left(h, h_{R}\right)
$$

with

$$
z(h, \psi)= \begin{cases}\frac{2 \sqrt{g_{z}}}{\sqrt{h}+\sqrt{\psi}} & \text { if } h \leq \psi \\ \sqrt{\frac{g_{z}}{2} \frac{h+\psi}{h \psi}} & \text { otherwise. }\end{cases}
$$

Complete solution. We have four different structural forms for the solution of the Riemann problem.

(1) If $h_{*}>\max \left\{h_{L}, h_{R}\right\}$, the solution is composed of a 1 -shock and a 2 -shock and reads

$$
\mathbf{W}(x, t)= \begin{cases}\mathbf{W}_{L}, & \text { if } x+\frac{m t^{2}}{2}<\dot{\sigma}_{1}\left(h_{L}, h_{*}, v_{L}\right) t \\ \mathbf{W}_{*}, & \text { if } \dot{\sigma}_{1}\left(h_{L}, h_{*}, v_{L}\right) t<x+\frac{m t^{2}}{2}<\dot{\sigma}_{2}\left(h_{*}, h_{R}, v_{*}\right) t, \\ \mathbf{W}_{R}, & \text { if } x+\frac{m t^{2}}{2}>\dot{\sigma}_{2}\left(h_{*}, h_{R}, v_{*}\right) t\end{cases}
$$

where the speeds of the discontinuities are

$$
\dot{\sigma}_{1}\left(h_{L}, h_{*}, v_{L}\right)=v_{L}+m t-h_{*} \sqrt{\frac{g_{z}}{2} \frac{h_{L}+h_{*}}{h_{L} h_{*}}}, \quad \dot{\sigma}_{2}\left(h_{R}, h_{*}, v_{R}\right)=v_{R}+m t+h_{*} \sqrt{\frac{g_{z}}{2} \frac{h_{*}+h_{R}}{h_{*} h_{R}}} .
$$

(2) If $h_{L}<h_{*}<h_{R}$, the solution is composed of a 1-shock and a 2-rarefaction and reads

$$
\mathbf{W}(x, t)= \begin{cases}\mathbf{W}_{L}, & \text { if } x+\frac{m t^{2}}{2}<\dot{\sigma}_{1}\left(h_{L}, h_{*}, v_{L}\right) t, \\ \mathbf{W}_{*}, & \text { if } \dot{\sigma}_{1}\left(h_{L}, h_{*}, v_{L}\right) t<x+\frac{m t^{2}}{2}<\left(v_{*}+m t+\sqrt{g_{z} h_{*}}\right) t, \\ \mathbf{W}_{2-\mathrm{rar}}, & \text { if }\left(v_{*}+m t+\sqrt{g_{z} h_{*}}\right) t<x+\frac{m t^{2}}{2}<\left(v_{R}+m t+\sqrt{g_{z} h_{R}}\right) t, \\ \mathbf{W}_{R}, & \text { if } x+\frac{m t^{2}}{2}>\left(v_{R}+m t+\sqrt{g_{z} h_{R}}\right) t,\end{cases}
$$

with

$$
\dot{\sigma}_{1}\left(h_{L}, h_{*}, v_{L}\right)=v_{L}+m t-h_{*} \sqrt{\frac{g_{z}}{2} \frac{h_{L}+h_{*}}{h_{L} h_{*}}}, \quad \mathbf{W}_{2-\mathrm{rar}}=\left(\begin{array}{c}
\frac{1}{9 g_{z}}\left(-v_{R}+2 \sqrt{g_{z} h_{R}}+\frac{x}{t}-\frac{m t}{2}\right)^{2} \\
\frac{1}{3}\left(v_{R}-2 \sqrt{g_{z} h_{R}}+2 \frac{x}{t}-m t\right)
\end{array}\right) .
$$

Note that, if $h_{L}=0$, it is natural to consider $v_{L}=0$ and then there is only a 2-rarefaction (the 1 -shock is not defined). In this case the solution reads

$$
\mathbf{W}(x, t)= \begin{cases}(0,0), & \text { if } x+\frac{m t^{2}}{2}<\left(v_{R}+m t-2 \sqrt{g_{z} h_{R}}\right) t \\ \mathbf{W}_{\text {rar }}, & \text { if }\left(v_{R}+m t-2 \sqrt{g_{z} h_{R}}\right) t<x+\frac{m t^{2}}{2}<\left(v_{R}+m t+\sqrt{g_{z} h_{R}}\right) t, \\ \mathbf{W}_{R}, & \text { if } x+\frac{m t^{2}}{2}>\left(v_{R}+m t+\sqrt{g_{z} h_{R}}\right) t .\end{cases}
$$

3 If $h_{R}<h_{*}<h_{L}$, the solution is composed of a 1-rarefaction and a 2 -shock and reads

$$
\mathbf{W}(x, t)= \begin{cases}\mathbf{W}_{L}, & \text { if } x+\frac{m t^{2}}{2}<\left(v_{L}+m t-\sqrt{g_{z} h_{L}}\right) t, \\ \mathbf{W}_{1-\text { rar }}, & \text { if }\left(v_{L}+m t-\sqrt{g_{z} h_{L}}\right) t<x+\frac{m t^{2}}{2}<\left(v_{*}+m t-\sqrt{g_{z} h_{*}}\right) t, \\ \mathbf{W}_{*}, & \text { if }\left(v_{*}+m t-\sqrt{g_{z} h_{*}}\right) t<x+\frac{m t^{2}}{2}<\dot{\sigma}_{2}\left(h_{R}, h_{*}, v_{R}\right) t, \\ \mathbf{W}_{R}, & \text { if } x+\frac{m t^{2}}{2}>\dot{\sigma}_{2}\left(h_{R}, h_{*}, v_{R}\right) t,\end{cases}
$$

with

$$
\dot{\sigma}_{2}\left(h_{R}, h_{*}, v_{R}\right)=v_{R}+m t+h_{*} \sqrt{\frac{g_{z}}{2} \frac{h_{*}+h_{R}}{h_{*} h_{R}}}, \quad \mathbf{W}_{1-\mathrm{rar}}=\left(\begin{array}{c}
\frac{1}{9 g_{z}}\left(v_{L}+2 \sqrt{g_{z} h_{L}}-\frac{x}{t}+\frac{m t}{2}\right)^{2} \\
\frac{1}{3}\left(v_{L}+2 \sqrt{g_{z} h_{L}}+2 \frac{x}{t}-m t\right)
\end{array}\right) .
$$


Again, if $h_{R}=0$, it is natural to consider $v_{R}=0$ and then there is only a 1-rarefaction (the 2 -shock is not defined). Then the solution reads

$$
\mathbf{W}(x, t)= \begin{cases}\mathbf{W}_{L}, & \text { if } x+\frac{m t^{2}}{2}<\left(v_{L}+m t-\sqrt{g_{z} h_{L}}\right) t \\ \mathbf{W}_{\text {rar }}, & \text { if }\left(v_{L}+m t-\sqrt{g_{z} h_{L}}\right) t<x+\frac{m t^{2}}{2}<\left(v_{L}+m t+2 \sqrt{g_{z} h_{L}}\right) t, \\ (0,0), & \text { if } x+\frac{m t^{2}}{2}>\left(v_{L}+m t+2 \sqrt{g_{z} h_{L}}\right) t .\end{cases}
$$

(4) If $h_{*}<\min \left\{h_{L}, h_{R}\right\}$, the solution is composed of a 1-rarefaction and a 2-rarefaction. We consider two cases:

- if $v_{R}-v_{L}<2 \sqrt{g}\left(\sqrt{h_{R}}+\sqrt{h_{L}}\right)$ (which ensure that $h_{*}>0$ ) the solution is

$$
\mathbf{W}(x, t)= \begin{cases}\mathbf{W}_{L}, & \text { if } x+\frac{m t^{2}}{2}<\left(v_{L}+m t-\sqrt{g_{z} h_{L}}\right) t, \\ \mathbf{W}_{1-\mathrm{rar}}, & \text { if }\left(v_{L}+m t-\sqrt{g_{z} h_{L}}\right) t<x+\frac{m t^{2}}{2}<\left(v_{*}+m t-\sqrt{g_{z} h_{*}}\right) t, \\ \mathbf{W}_{*}, & \text { if }\left(v_{*}+m t-\sqrt{g_{z} h_{*}}\right) t<x+\frac{m t^{2}}{2}<\left(v_{*}+m t+\sqrt{g_{z} h_{*}}\right) t, \\ \mathbf{W}_{2-\text { rar }}, & \text { if }\left(v_{*}+m t+\sqrt{g_{z} h_{*}}\right) t<x+\frac{m t^{2}}{2}<\left(v_{R}+m t+\sqrt{g_{z} h_{R}}\right) t, \\ \mathbf{W}_{R}, & \text { if } x+\frac{m t^{2}}{2}>\left(v_{R}+m t+\sqrt{g_{z} h_{R}}\right) t\end{cases}
$$

- if the initial states do not satisfy the condition $v_{R}-v_{L}>2 \sqrt{g}\left(\sqrt{h_{R}}+\sqrt{h_{L}}\right)$, the Riemann problem has no solution in the above sense. However, one can yet define a solution by introducing a dry state and the solution consists of two rarefaction waves separated by a dry zone where $h=0$ and the other dependent variables are left undefined (classically one set $v=0)$

$$
\mathbf{W}(x, t)= \begin{cases}\mathbf{W}_{L}, & \text { if } x+\frac{m t^{2}}{2}<\left(v_{L}+m t-\sqrt{g_{z} h_{L}}\right) t, \\ \mathbf{W}_{1-\text { rar }}, & \text { if }\left(v_{L}+m t-\sqrt{g_{z} h_{L}}\right) t<x+\frac{m t^{2}}{2}<\left(v_{L}+m t+2 \sqrt{g_{z} h_{L}}\right) t, \\ (0,0), & \text { if } \left.v_{L}+m t+2 \sqrt{g_{z} h_{L}}\right) t<x+\frac{m t^{2}}{2}<\left(v_{R}+m t-2 \sqrt{g_{z} h_{R}}\right) t, \\ \mathbf{W}_{2-\text { rar }}, & \text { if }\left(v_{R}+m t-2 \sqrt{g_{z} h_{R}}\right) t<x+\frac{m t^{2}}{2}<\left(v_{R}+m t+\sqrt{g_{z} h_{R}}\right) t, \\ \mathbf{W}_{R}, & \text { if } x+\frac{m t^{2}}{2}>\left(v_{R}+m t+\sqrt{g_{z} h_{R}}\right) t,\end{cases}
$$

with

$$
\mathbf{W}_{1-\mathrm{rar}}=\left(\begin{array}{c}
\frac{1}{9 g_{z}}\left(v_{L}+2 \sqrt{g_{z} h_{L}}-\frac{x}{t}+\frac{m t}{2}\right)^{2} \\
\frac{1}{3}\left(v_{L}+2 \sqrt{g_{z} h_{L}}+2 \frac{x}{t}-m t\right)
\end{array}\right), \quad \mathbf{W}_{2-\mathrm{rar}}=\left(\begin{array}{c}
\frac{1}{9 g_{z}}\left(-v_{R}+2 \sqrt{g_{z} h_{R}}+\frac{x}{t}-\frac{m t}{2}\right)^{2} \\
\frac{1}{3}\left(v_{R}-2 \sqrt{g_{z} h_{R}}+2 \frac{x}{t}-m t\right)
\end{array}\right) .
$$

\section{A.4. Entropy dissipation}

For this system, the total energy $E$ and the energy flux $G$ are respectively

$$
E=\frac{h(v+m t)^{2}+g_{z} h^{2}}{2}, \quad G=\left(\frac{h(v+m t)^{2}}{2}+g_{z} h^{2}\right)(v+m t) .
$$

We can compute

$$
\frac{\partial E}{\partial \mathbf{W}}=\left(\begin{array}{c}
\frac{(v+m t)^{2}}{2}+g_{z} h \\
h(v+m t)
\end{array}\right), \quad \frac{\partial G}{\partial \mathbf{W}}=\left(\begin{array}{c}
\left(\frac{(v+m t)^{2}}{2}+2 g_{z} h\right)(v+m t) \\
\frac{3}{2} h(v+m t)^{2}+g_{z} h^{2}
\end{array}\right),
$$

thus $\frac{\partial E}{\partial \mathbf{W}}\left(\frac{\partial \mathbf{V}}{\partial \mathbf{W}}\right)^{-1} \frac{\partial F}{\partial \mathbf{W}}=\frac{\partial G}{\partial \mathbf{W}}$. This says that the total energy function is an entropy function for the system (5). To see whether the total energy function is convex, we compute the matrix of second derivatives:

$$
\frac{\partial}{\partial \mathbf{W}}\left(\frac{\partial E}{\partial \mathbf{W}}\right)^{T}=\left(\begin{array}{cc}
g_{z} & v+m t \\
v+m t & h
\end{array}\right) .
$$


This matrix is positive defined if and only if $(v+m t)^{2}<g h$.

Let $\left(h_{\ell}, v_{\ell}\right)$ and $\left(h_{r}, v_{r}\right)$ two states and let us note $\llbracket \star \rrbracket \equiv \star_{r}-\star_{\ell}$. Recalling that at propagating discontinuity the Rankine-Hugoniot conditions (8) require $\llbracket h(v+m t) \rrbracket=\llbracket h \rrbracket \dot{\sigma}_{k}$ and $\llbracket h v(v+m t)+\frac{g_{z}}{2} h^{2} \rrbracket=\llbracket h v \rrbracket \dot{\sigma}_{k}$, it follows that

$$
\begin{aligned}
\llbracket E \rrbracket & =\frac{1}{2} \llbracket h(v+m t)^{2}+g_{z} h^{2} \rrbracket=\frac{1}{2} \llbracket h v(v+m t)+g_{z} h^{2}+h(v+m t) m t \rrbracket \\
& =\frac{1}{2} \llbracket h v(v+m t)+\frac{g_{z}}{2} h^{2} \rrbracket+\frac{g_{z}}{4} \llbracket h^{2} \rrbracket+\frac{1}{2} \llbracket h(v+m t) \rrbracket m t \\
& =\frac{1}{2} \llbracket h v \rrbracket \dot{\sigma}_{k}+g_{z} \frac{\left(h_{\ell}+h_{r}\right)}{4} \llbracket h \rrbracket+\frac{1}{2} \llbracket h \rrbracket \dot{\sigma}_{k} m t \\
& =\frac{1}{2}(\llbracket h(v+m t) \rrbracket-\llbracket h \rrbracket m t) \dot{\sigma}_{k}+g_{z} \frac{\left(h_{\ell}+h_{r}\right)}{4} \llbracket h \rrbracket+\frac{1}{2} \llbracket h \rrbracket \dot{\sigma}_{k} m t \\
& =\frac{1}{2} \llbracket h \rrbracket\left(\dot{\sigma}_{k}^{2}+g_{z} \frac{\left(h_{\ell}+h_{r}\right)}{2}\right) .
\end{aligned}
$$

For a 1-shock, the Lax admissibility condition requires that $\llbracket h \rrbracket>0$, which implies $\llbracket E \rrbracket>0$. In this case, the velocities of the flow relative to the shock are

$$
\left(v_{L}+m t\right)-\dot{\sigma}_{1}=\sqrt{g_{z} \frac{h_{L}+h_{*}}{2} \frac{h_{*}}{h_{L}}}, \quad\left(v_{*}+m t\right)-\dot{\sigma}_{1}=\sqrt{g_{z} \frac{h_{L}+h_{*}}{2} \frac{h_{L}}{h_{*}}} .
$$

In both cases, the velocity of the flow relative to the shock is positive. Thus in this case the total energy $E$ decreases from the pre-shock state (the intermediate state) to the post-shock state (the left state). Similarly, for a 2 -shock we have $\llbracket h \rrbracket<0$ and then $\llbracket E \rrbracket<0$ and the velocities of the flow relative to the shock are:

$$
\left(v_{*}+m t\right)-\dot{\sigma}_{2}=-\sqrt{g_{z} \frac{h_{R}+h_{*}}{2} \frac{h_{R}}{h_{*}}}, \quad\left(v_{R}+m t\right)-\dot{\sigma}_{2}=-\sqrt{g_{z} \frac{h_{R}+h_{*}}{2} \frac{h_{*}}{h_{R}}} .
$$

On both sides of the shock the velocity of the flow relative to the shock is negative, so this condition says that the total energy $E$ decreases from the pre-shock state (the intermediate state) to the postshock state (the right state).

\section{REFERENCES}

[1] Ancey, C., Iverson, R. M., Rentschler, M., and Denlinger, R. P. (2008). An exact solution for ideal dam-break floods on steep slopes. Water Resources Research, 44(1):1-10.

[2] Bouchut, F., Fernández-Nieto, E. D., Mangeney, A., and Lagrée, P. Y. (2008). On new erosion models of savage-hutter type for avalanches. Acta Mechanica, 199(1):181-208.

[3] Crosta, G. B., Imposimato, S., and Roddeman, D. (2009a). Numerical modeling of 2-d granular step collapse on erodible and nonerodible surface. J. Geophys. Res, 114:F03020.

[4] Crosta, G. B., Imposimato, S., and Roddeman, D. (2009b). Numerical modelling of entrainment/deposition in rock and debris-avalanches. Engineering geology, 109(1-2):135-145.

[5] Deboeuf, S., Dauchot, O., Staron, L., Mangeney, A., and Vilotte, J. P. (2005). Memory of the unjamming transition during cyclic tiltings of a granular pile. Phys. Rev. E., 72.

[6] Dressler, R. F. (1958). Unsteady non-linear waves in damping channels. Journal of the National Bureau of Standards, 2(3):217-225.

[7] Farin, M., Mangeney, A., Roche, O., Ionescu, I., and Hungr, O. (2011). Landslide boost from entrainment of erodible material along the slope. In AGU Fall Meeting Abstracts.

[8] Favreau, P., Mangeney, A., Lucas, A., Crosta, G., and Bouchut, F. (2010). Numerical modeling of landquakes. Geophys. Res. Lett, 37:L15305.

[9] Fernandez-Feria, R. (2006). Dam-break flow for arbitrary slopes of the bottom. Journal of engineering mathematics, 54(4):319-331.

[10] Godlewski, E. and Raviart, P.-A. (1996). Numerical Approximation of Hyperbolic Systems of Conservation Laws, volume 118. Springer.

[11] Gray, J. and Ancey, C. (2009). Segregation, recirculation and deposition of coarse particles near two-dimensional avalanche fronts. Journal of Fluid Mechanics, 629(1):387-423. 
[12] Hibert, C., Mangeney, A., Grandjean, G., and Shapiro, N. (2011). Slopes instabilities in the Dolomieu crater, la réunion island: from the seismic signal to the rockfalls characteristics. $J$. Geophys. Res., in press:-.

[13] Hogg, A. J. (2006). Lock-release gravity currents and dam-break flows. Journal of Fluid Mechanics, 553(569):61-87.

[14] Hungr, O. and Evans, S. G. (2004). Entrainment of debris in rock avalanches: An analysis of a long run-out mechanism. Bulletin of the Geological Society of America, 116(9-10):1240.

[15] Iverson, R. M., Reid, M. E., Logan, M., LaHusen, R. G., Godt, J. W., and Griswold, J. P. (2011). Positive feedback and momentum growth during debris-flow entrainment of wet bed sediment. Nat. Geosci., 4:116-121.

[16] Karelsky, K. V., Papkov, V. V., and Petrosyan, A. S. (2000a). The initial discontinuity decay problem for shallow-water equations on slopes. Physics Letters A, 271(5-6):349-357.

[17] Karelsky, K. V., Papkov, V. V., Petrosyan, A. S., and Tsygankov, D. V. (2000b). Particular solutions of shallow-water equations over a non-flat surface. Physics Letters A, 271(5-6):341348.

[18] Kerswell, R. R. (2005). Dam break with coulomb friction: A model for granular slumping? Physics of Fluids, 17:057101.

[19] Kuo, C. Y., Tai, Y. C., Bouchut, F., Mangeney, A., Pelanti, M., Chen, R. F., and Chang, K. J. (2009). Simulation of tsaoling landslide, taiwan, based on saint venant equations over general topography. Engineering Geology, 104(3-4):181-189.

[20] Lacaze, L., Phillips, J. C., and Kerswell, R. R. (2008). Planar collapse of a granular column: Experiments and discrete element simulations. Physics of Fluids, 20(6):063302-063302.

[21] LeVeque, R. J. (1994). Numerical Methods for Conservation Laws. Birkhäuser Verlag AG.

[22] Lube, G., Huppert, F. E., Sparks, R. S., and Freundt, A. (2011). Granular column collapses down rough, inclined channels. Journal of Fluid Mechanics, 675:347-368.

[23] Lucas, A. and Mangeney, A. (2007). Mobility and topographic effects for large valles marineris landslides on mars. Geophysical research letters, 34(10):L10201.

[24] Lucas, A., Mangeney, A., Mège, D., and Bouchut, F. (2011). Influence of the scar geometry on landslide dynamics and deposits: Application to martian landslides. J. Geophys. Res. - Planets, 116.

[25] Mallogi, F., Lanuza, J., Andreotti, B., and Clement, E. (2006). Erosion waves: Transverse instabilities and fingering. Europhys. Lett., 75:825.

[26] Mangeney, A. (2011). Geomorphology : Landslide boost from entrainment. Nature Geoscience, 4:77-78.

[27] Mangeney, A., Bouchut, F., Thomas, N., Vilotte, J. P., and Bristeau, M. O. (2007a). Numerical modeling of self-channeling granular flows and of their levee-channel deposits. Journal of Geophysical Research - all series -, 112(F2):2017.

[28] Mangeney, A., Heinrich, P., and Roche, R. (2000). Analytical solution for testing debris avalanche numerical models. Pure and Applied Geophysics, 157(6-8):1081-1096.

[29] Mangeney, A., Roche, O., Hungr, O., Mangold, N., Faccanoni, G., and Lucas, A. (2010). Erosion and mobility in granular collapse over sloping beds. J. Geophys. Res, 115:F03040.

[30] Mangeney, A., Staron, L., Volfson, D., and Tsimring, L. (2006). Comparison between discrete and continuum modeling of granular spreading. WSEAS Trans. Math, 2(6):373-380.

[31] Mangeney, A., Tsimring, L. S., Volfson, D., Aranson, I. S., and Bouchut, F. (2007b). Avalanche mobility induced by the presence of an erodible bed and associated entrainment. Geophys. Res. Lett, 34(10.1029).

[32] Mangeney-Castelnau, A., Bouchut, F., Vilotte, J. P., Lajeunesse, E., Aubertin, A., and Pirulli, M. (2005). On the use of saint venant equations to simulate the spreading of a granular mass. $J$. Geophys. Res, 110:B09103.

[33] Mangeney-Castelnau, A., Vilotte, J. P., Bristeau, M. O., Perthame, B., Bouchut, F., Simeoni, C., and Yerneni, S. (2003). Numerical modeling of avalanches based on saint venant equations using a kinetic scheme. J. Geophys. Res, 108(2527):4.

[34] McDougall, S. and Hungr, O. (2004a). A model for the analysis of rapid landslide motion 
across three-dimensional terrain. Canadian Geotechnical Journal, 41(6):1084-1097.

[35] McDougall, S. and Hungr, O. (2004b). A universal model for 3-d runout analysis of landslides. In Proceedings, 9th International Symposium on Landslides, Rio de Janeiro, Brazil.

[36] Pirulli, M. and Mangeney, A. (2008). Results of back-analysis of the propagation of rock avalanches as a function of the assumed rheology. Rock Mechanics and Rock Engineering, 41(1):59-84.

[37] Pouliquen, O. and Forterre, Y. (2002). Friction law for dense granular flows: application to the motion of a mass down a rough inclined plane. Journal of Fluid Mechanics, 453:133-151.

[38] Roche, O., Attali, M., Mangeney, A., and Lucas, A. (2011). On the run out distance of geophysical gravitational flows: insight from fluidized granular collapse experiments. Earth Planet. Sci. Lett., 311:375-385.

[39] Savage, S. B. and Hutter, K. (1989). The motion of a finite mass of granular material down a rough incline. Journal of Fluid Mechanics, 199(1):177-215.

[40] Sovilla, B., Bartelt, P., et al. (2002). Observations and modelling of snow avalanche entrainment.

[41] Sovilla, B., Burlando, P., and Bartelt, P. (2006). Field experiments and numerical modeling of mass entrainment in snow avalanches. Journal of geophysical research, 111(F3):F03007.

[42] Stoker, J. J. (1992). Water waves: the mathematical theory with applications, volume 36. Wiley-Interscience.

[43] Toro, E. F. (2009). Riemann solvers and numerical methods for fluid dynamics. SpringerVerlag, Berlin, third edition. A practical introduction.

[44] Trangenstein, J. A. (2009). Numerical solution of hyperbolic partial differential equations. Cambridge Univ Press.

[45] Ungarish, M. (2011). Two-layer shallow-water dam-break solutions for non-boussinesq gravity currents in a wide range of fractional depth. Journal of Fluid Mechanics, 675(1):2759.

[46] Voivret, C., Radjai, F., Delenne, J., and El Youssoufi, M. (2007). Space-filling properties of polydisperse granular media. Physical Review E, 76(2):021301.

[47] Zhao, C., Hobbs, B. E., and Ord, A. (2009). Fundamentals of Computational Geoscience: Numerical Methods and Algorithms, volume 122. Springer Verlag.

[48] Zhao, C., Hobbs, B. E., and Ord, A. (2010). Theoretical analyses of nonaqueous phase liquid dissolution-induced instability in two-dimensional fluid-saturated porous media. International Journal for Numerical and Analytical Methods in Geomechanics, 34(17):1767-1796. 